\title{
In-situ X-ray radiography of primary Fe-rich intermetallic compound formation
}

\author{
Shikang Feng ${ }^{* 1}$, Enzo Liotti ${ }^{1}$, Andrew Lui ${ }^{1}$, Matthew D. Wilson ${ }^{2}$, \\ Thomas Connolley $^{3}$, Ragnvald H. Mathiesen ${ }^{4}$, and Patrick S. Grant ${ }^{1}$ \\ ${ }^{1}$ Department of Materials, University of Oxford, Oxford, OX1 3PH, UK \\ ${ }^{2}$ Science and Technology Facilities Council, Rutherford Appleton Laboratory, Harwell Science \\ and Innovation Campus, Didcot, OX11 ODE, UK \\ ${ }^{3}$ Diamond Light Source, Harwell Science and Innovation Campus, Didcot, OX11 ODE, UK \\ ${ }^{4}$ Department of Physics, Norwegian University of Science and Technology (NTNU), N-7491 \\ Trondheim, Norway
}

\begin{abstract}
The formation of primary Fe-rich intermetallic compound crystals and the effect of $\mathrm{TiB}_{2}$ and $\mathrm{TiC}$ inoculation during solidification of a hypereutectic Al-Fe alloy has been investigated. Both horizontal, near isothermal conditions and vertical, directional thermal gradient conditions were studied using laboratory and synchrotron $\mathrm{X}$-ray radiography. $\mathrm{TiB}_{2}$ and $\mathrm{TiC}$ inoculation enhanced formation of Fe-rich intermetallics under all experimental conditions. Near isothermal solidification resulted in the highest intermetallic number density and average formation rate, whilst increasing the thermal gradient reduced both. A model for intermetallic crystal formation is proposed to explain the effect of thermal gradient and cooling rate on the intermetallic number density and average formation rate.
\end{abstract}

Keywords: Fe-rich intermetallics; Crystal formation; Inoculation; In-situ Xradiography; Al alloys

\section{Introduction}

Secondary Al production by recycling of end-of-life $\mathrm{Al}$ alloy scrap is a well-established route that saves up to $95 \%$ of energy consumption and over $90 \%$ of greenhouse gas emission when compared with primary $\mathrm{Al}$ production from bauxite [1]. However, the accumulation of impurities, Fe in particular, hinders more extensive use of $\mathrm{Al}$

\footnotetext{
*Corresponding author.

Email address: shikang.feng@materials.ox.ac.uk (S. Feng).
} 
recycling, especially for wrought alloys with tight compositional tolerances [2]. Because of the large difference between the liquid solubility $\left(2.5 \mathrm{wt} . \%\right.$ at $700^{\circ} \mathrm{C}$ and 5 wt. $\%$ at $800^{\circ} \mathrm{C}$ ) and solid solubility (less than 0.05 wt.\%) of Fe in Al [3], Fe precipitates during solidification and generally form relatively coarse (typically in the range of $\sim 50 \mu \mathrm{m}$ to several millimetres [4]), anisotropic plate-like (or needle-like in 2D) Fe-rich intermetallic compounds (IMCs), e.g. $\mathrm{Al}_{13} \mathrm{Fe}_{4}$ and $\beta-\mathrm{Al}_{5} \mathrm{FeSi}$. These IMCs may act as stress concentrators and in particular degrade the ductility and toughness of the alloy [5, 6]. Current strategies for controlling and mitigating the potentially damaging effects of these Fe-rich IMCs involve diluting the recycled $\mathrm{Al}$ alloy feedstock with virgin smelted $\mathrm{Al}$ produced from energy-intensive primary production [7], which consumes 66 MJ energy for every $1 \mathrm{~kg}$ of $\mathrm{Al}$ [8]. Relatively long (in the order of days $[9,10]$ ) high temperature treatments and thermomechanical processing are also used to refine IMCs produced by solidification, at considerable additional cost.

Consequently significant effort has been expended to control better the morphology, size and distribution of Fe-rich IMCs during the solidification stage by understanding their nucleation, growth and phase selection behaviour in the hope that higher concentrations of Fe (and other "tramp" elements) might be tolerated [11 22]. Particular effort has been spent in exploring potential nucleants for Ferich IMCs. The majority of these studies have been based on post-solidification microscopy, with sometimes contradictory findings. Samuel et al. 23] reported an increased number of $\beta-\mathrm{Al}_{5} \mathrm{FeSi}$ IMCs upon addition of $\mathrm{P}$, which formed $\mathrm{AlP}$ particles with molten $\mathrm{Al}$, and concluded that $\mathrm{AlP}$ promoted the nucleation of $\beta$ - $\mathrm{Al}_{5} \mathrm{FeSi}$. In contrast, Lu and Dahle 24] found no increase in the number of $\beta-\mathrm{Al}_{5} \mathrm{FeSi}$ platelets upon $\mathrm{P}$ addition, and no evidence for physical contact between the IMC platelets and AlP particles. Cao and Campbell [25, 26] suggested that Fe-rich IMCs can nucleate on the outer surface of oxide bi-films. Similarly, Miller et al. [27] and Narayanan et al. 28] also suggested nucleation of Fe-rich IMCs on oxides, by observation of direct contact. Khalifa et al. [18] performed extensive metallography on Al-Si-Fe alloys inoculated with various types of particles including $\alpha-\mathrm{Al}_{2} \mathrm{O}_{3}$ powder, 
TiC powder and $\mathrm{TiB}_{2}$ powder. They examined the potency of each type of particle by quantifying the number fraction of powder particles in contact with Fe-rich IMC plates in each condition and found that the number fraction of particles in contact with the IMCs showed a weak relationship with the particle type. It was concluded that nucleation of platelet Fe-rich IMCs was not strongly influenced by the type of particle addition [18]. Extensive IMC extraction and microscopy by Lui et al. [29] suggested that $\mathrm{TiB}_{2}$ from the addition of commercial Al-5Ti-1B might be associated with nucleation of secondary Fe-rich IMCs, evidenced by observation of $\mathrm{TiB}_{2}$ particles in physical contact with IMC platelets after extraction.

More recently, in-situ X-ray radiography and tomography have increasingly been used to study Fe-rich IMCs for a better understanding of the early stages of IMC formation and growth. However, only a handful of experiments resolved sufficiently early stage formation to gain insight of nucleation [30, 31]; most studies have focused on growth and phase selection $[32[36]$.

The early stage formation of secondary IMCs is especially difficult to study in real-time, primarily due to their low volume fraction (volume fraction $<2 \%$ ) compared with the presence of a much greater fraction of pre-formed, primary $\alpha$-Al dendrites (volume fraction $>70 \%$ ). An in-situ tomography study of $\mathrm{Al}-8 \mathrm{Si}-4 \mathrm{Cu}-$ $0.8 \mathrm{Fe}$ (wt.\%) at a cooling rate of $0.02 \mathrm{Ks}^{-1}$ by Terzi et al. [30] resolved the formation of four secondary $\beta$-IMCs, all of which were associated with the surface oxide skin of the sample. Later, Puncreobutr et al. 31] performed a more systematic in-situ tomography study on $\mathrm{Al}-7.5 \mathrm{Si}-3.5 \mathrm{Cu}-0.6 \mathrm{Fe}$ (wt.\%) at $0.05 \mathrm{Ks}^{-1}$ and also observed some secondary Fe-rich IMCs near or at the sample oxide skin, whilst the majority of IMCs $(>60 \%)$ were in contact with the primary $\alpha$-Al dendrites, presumably due to physical impingement of growing IMCs with pre-formed $\alpha$-Al dendrites. Overall, the low volume fraction of secondary phase IMCs in commercial-type alloys presents significant practical difficulties for X-ray radiography or tomography experiments.

In this work, a hypereutectic Al-Fe alloy in which plate-like Fe-rich IMCs $\left(\mathrm{Al}_{13} \mathrm{Fe}_{4}\right)$ formed as primary phase was chosen. Formation of primary Fe-rich IMCs with different inoculation conditions (no inoculation, $\mathrm{TiB}_{2}$ and $\mathrm{TiC}$ ) was studied at a series 
of cooling rates and thermal gradients. Support is provided from a large number of measurements (4,531 IMCs in total) that inoculation directly enhanced the formation of primary Fe-rich IMCs, and under some conditions, the internal surface of oxide skin around the sample also catalysed nucleation. An increase in thermal gradient is shown to reduce significantly the number density and average formation rate of primary IMCs, and also to decrease their formation temperature. A model is proposed to explain these trend-wise variations, accounting for the effect of thermal gradient and cooling rate, in terms of undercooled liquid volume and maximum undercooling available within the volume.

\section{Experimental methods}

\subsection{Sample preparation}

An alloy composition of $\mathrm{Al}-3 \mathrm{Fe}$ (wt\%) was chosen to form plate-like $\mathrm{Al}_{13} \mathrm{Fe}_{4}$ IMCs as primary phase directly from the liquid, allowing their formation behaviour to be studied without constraint due to the presence of any prior $\mathrm{Al}$ grains. Alloy samples were prepared following a four-step procedure. First pure $\mathrm{Al}(99.999 \%)$ and an $\mathrm{Al}-$ $75 \mathrm{Fe}(\mathrm{wt} \%)$ master alloy were fully melted and mixed by holding at $850^{\circ} \mathrm{C}$ for 30 min in an enclosed induction furnace under a controlled positive Ar pressure (to restrict evaporation and oxidation of $\mathrm{Al}$ ) and then left to air cool. Second, the "green" alloy obtained from the first step was remelted in a clay graphite crucible at $750^{\circ} \mathrm{C}$, carefully stirred and cast into a water-cooled $\mathrm{Cu}$ mould containing 3 cylindrical cavities of $30 \mathrm{~mm}$ diameter and $40 \mathrm{~mm}$ depth, to obtain 3 cylindrical ingots. This step was carried out before casting the alloy into the final shape in order to reduce possible macro-segregation caused by slower cooling in the first step. Third, each of the 3 cylindrical ingots were remelted again and with different inoculant conditions: no inoculant addition, $0.5 \mathrm{wt} \%$ Al-5Ti-1B master alloy, and $0.5 \mathrm{wt} \% \mathrm{Al}-5 \mathrm{Ti}-0.2 \mathrm{C}$ master alloy. The well-mixed melt was then poured into a rectangular $\mathrm{Cu}$ mould with an internal cavity of $55 \times 8 \times 16 \mathrm{~mm}$, following the design 
in [37]. The combination of small casting geometry and fast cooling effectively eliminated any significant macro-segregation [37]. Fig. 1 shows a schematic of the casting arrangement that promoted rapid mould filling; the location of the foil samples used for radiography experiments is also indicated. After the upper and lower parts of the casting were removed, foil samples were produced using wire electro-discharge machining (wire EDM), as shown in Fig. 1.
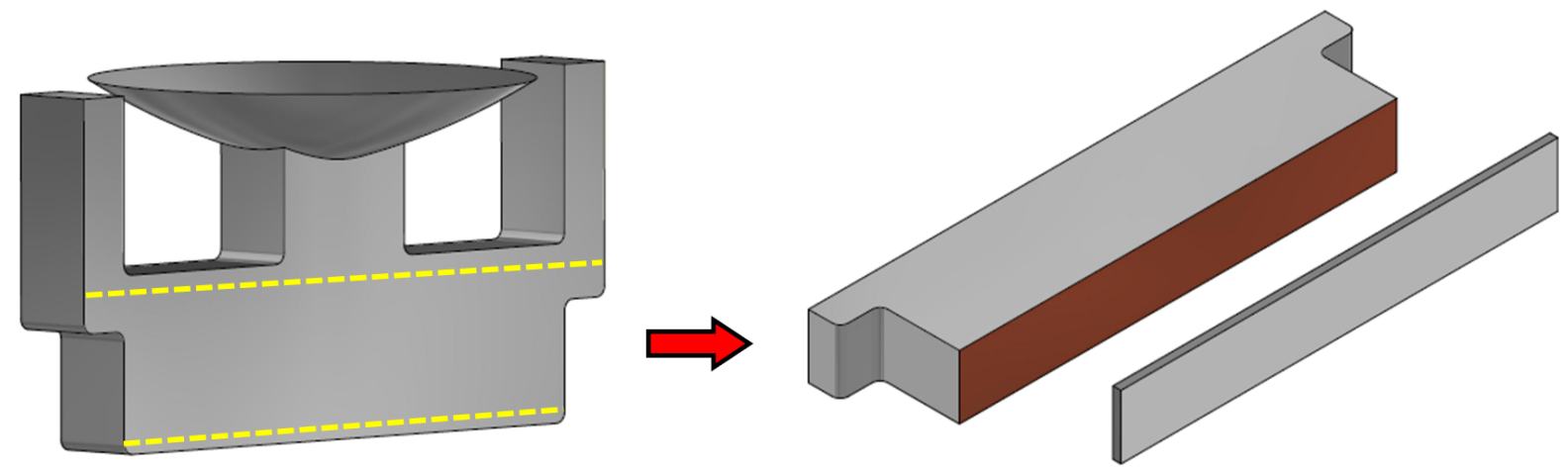

Figure 1: A schematic showing the casting arrangement and how foil samples were sectioned from the bulk Al-Fe ingot. The as-cast ingot was sectioned as indicated by the yellow dashed lines, and sliced using wire electro-discharge machining (wire EDM) to produce foil samples.

Finally, X-ray radiography samples of $50 \times 5 \mathrm{~mm}$ and thickness of $190 \pm 5 \mu \mathrm{m}$ were prepared by grinding and polishing to a final surface finish of $1 \mu \mathrm{m}$. Each sample was encapsulated in an X-ray transparent glassy carbon cell (Sigradur K) of $100 \mu \mathrm{m}$ thickness. For convenience, the Al-3Fe (wt\%) alloy samples with different inoculation conditions are named: AF (no inoculant), AF-B (with $\mathrm{TiB}_{2}$ ) and AF-C (with TiC). Overall, 10 samples were prepared: 4 AF, 4 AF-B and 2 AF-C.

\subsection{In-situ X-ray radiography}

In-situ X-ray radiography investigations were carried out using both synchrotron $\mathrm{X}$-rays for vertical, directional solidification, and laboratory X-rays for horizontal, near-isothermal solidification, as shown in Figs. 2(a) and (b) respectively. Horizontal solidification offered the advantage of significantly reduced liquid convection, similar to micro-gravity conditions [38], and is a configuration generally not available at synchrotrons. On the other hand, the synchrotron experiments provided a better contrast and a higher spatial and temporal resolution. Table 1 summarises 
the key parameters of the two experimental arrangements, and Table 2 lists all the experimental conditions, including the number of repeats and the number of IMC crystals measured in each condition. Overall, 56 experiments were performed and 4,531 IMC crystals measured.
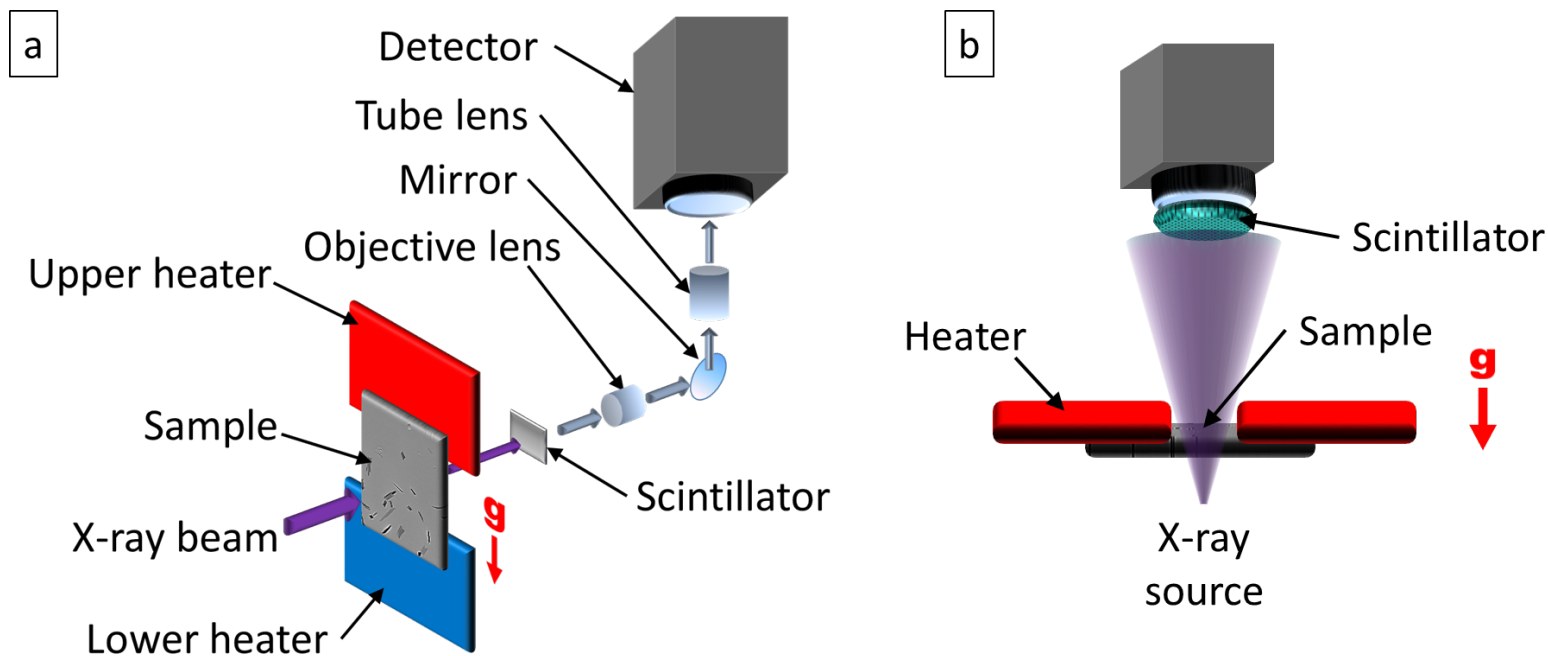

Figure 2: Schematic of the experimental configurations for (a) synchrotron radiography experiments where gravity was parallel to the sample surface, and the incident X-ray was a parallel beam, and (b) laboratory radiography experiments where gravity was perpendicular to the sample surface, and the incident X-ray was a cone beam.

\begin{tabular}{|c|c|c|c|c|c|c|c|c|}
\hline & Source & Beam & $\begin{array}{l}\text { Peak energy } \\
(\mathrm{keV})\end{array}$ & Scintillator & Camera & $\begin{array}{c}\text { Temporal resolution } \\
(\mathrm{Hz})\end{array}$ & $\begin{array}{l}\text { Image pixel size } \\
(\mu \mathrm{m} / \text { pixel })\end{array}$ & $\begin{array}{c}\mathrm{FoV} \\
\left(\mathrm{mm}^{2}\right)\end{array}$ \\
\hline Laboratory & Mo micro-focus & $\mathrm{C}, \mathrm{W}$ & 17.4 & CsI & Vosskuhler DXI-11000 & 2 & $1.5(5)^{1}$ & $3 \times 1.8$ \\
\hline Synchrotron & Undulator & $\mathrm{P}, \mathrm{W}$ & 19 & LuAG:Ce & PCO.Edge & 10 & 2.2 & $4.5 \times 3$ \\
\hline
\end{tabular}

Table 1: A summary of the set-up of the two radiography configurations, using a laboratory apparatus at Norwegian University of Science and Technology (NTNU), Norway, and using synchrotron source at beamline ID19, European Synchrotron Radiation Facility (ESRF), France. C = cone beam, P = parallel beam, $\mathrm{W}=$ white beam. ${ }^{1}$ The value in the parentheses is the ultimate spatial resolution.

\subsubsection{Laboratory X-ray radiography}

Laboratory in-situ X-ray radiography experiments were conducted using a bespoke laboratory apparatus at the Norwegian University of Science and Technology (NTNU), Norway. The arrangement comprised a microfocus Mo-anode X-ray tube, a Bridgman solidification furnace (XRMON-GF), and a CCD camera (Vosskuhler DXI11000) with a Scint-X CsI scintillator. A more detailed description of the apparatus can be found in [37-42]. The tube voltage and tube current were adjusted 


\begin{tabular}{|c|c|c|c|c|c|c|}
\hline \multirow[t]{2}{*}{ Alloy } & \multirow[t]{2}{*}{ Direction } & \multirow[t]{2}{*}{ Thermal gradient $\left(\mathrm{K} \mathrm{mm}^{-1}\right)$} & \multicolumn{4}{|c|}{ Cooling rate $\left(\mathrm{Ks}^{-1}\right)$} \\
\hline & & & 0.5 & 1 & 2 & 4 \\
\hline $\mathrm{AF}$ & $\mathrm{h}$ & & $4(113)$ & $4(181)$ & $4(289)$ & - \\
\hline AF-B & $\mathrm{h}$ & $\approx 0$ & $3(121)$ & $3(362)$ & $3(545)$ & - \\
\hline $\mathrm{AF}-\mathrm{C}$ & $\mathrm{h}$ & & $3(116)$ & $4(301)$ & $6(610)$ & - \\
\hline $\mathrm{AF}$ & $\mathrm{v}$ & 5 & $2(159)$ & $2(188)$ & $2(212)$ & $2(201)$ \\
\hline AF-B & $\mathrm{v}$ & 5 & $2(258)$ & $1(98)$ & $2(288)$ & $2(256)$ \\
\hline AF-B & $\mathrm{v}$ & 8 & $1(29)$ & $2(67)$ & $2(58)$ & $2(79)$ \\
\hline
\end{tabular}

Table 2: A summary of all the experimental conditions, showing the number of repeats and the number of IMC crystals measured (in parenthesis) in each experimental condition. Overall 56 solidification experiments were carried out, 34 in a horizontal near-isothermal condition using laboratory apparatus and 22 in a vertical directional condition at a synchrotron. 4,531 crystals were studied in total. $\mathrm{h}=$ horizontal and $\mathrm{v}=$ vertical.

to $50 \mathrm{kV}$ and $100 \mu \mathrm{A}$ to maximise absorption contrast between the Fe-rich IMC paricles and the Al-rich liquid, and the sample-source and sample-detector distance were also set for the best compromise between magnification and frame rate. Radiography videos were recorded at $2 \mathrm{~Hz}$ with an image pixel size of approximately 1.5 $\mu \mathrm{m} /$ pixel. The image pixel size was calculated by moving the sample up or down by an accurately known distance via the control module while counting its displacement in pixels from the radiography video. The ultimate spatial resolution (i.e. the smallest feature able to be resolved, calculated according to [39]) as a function of the source spot size, scintillator resolution and magnification was estimated to be approximately $5 \mu \mathrm{m}$.

Experiments were performed in a $\mathrm{N}_{2}$ atmosphere with an $\mathrm{O}_{2}$ concentration measured consistently $<1 \%$, to protect both the sample and the glassy carbon cell from excessive oxidation. During the experiment, each sample was set in a horizontal position, i.e. with the flat surface of the sample perpendicular to gravity. Each sample was fully melted and held at $750^{\circ} \mathrm{C}$ for $3 \mathrm{~min}$, following which it was cooled in a near-isothermal condition at a constant cooling rate ranging from $0.5 \mathrm{Ks}^{-1}$ to

IMC particles were studied (see Table 2). 


\subsubsection{Synchrotron X-ray radiography}

In-situ synchrotron X-ray radiography experiments were carried out at beamline ID19, European Synchrotron Radiation Facility (ESRF), France, using a bespoke solidification rig designed and built for synchrotron X-ray radiography studies. The rig contained two vertically aligned poly-graphite plate heaters encapsulated in polyBN. Each sample cell was mounted onto a spring steel sample holder using high temperature cement and attached in close contact with both plate heaters. The chamber utilised a $25 \mu \mathrm{m}$ thick X-ray transparent Kapton thermal insulating film as the window material in the X-ray path. The solidification rig was controlled remotely via a National Instruments module connected to a PC. The temperature of the two heaters was controlled independently by a PID algorithm coded in LabView, with the temperature of each heater monitored by a K-type thermocouple mounted in the centre of the heating zone at the back of the heater. The maximum achievable cooling rate and temperature of the heaters were $28 \mathrm{Ks}^{-1}$ and $1400^{\circ} \mathrm{C}$ respectively. The rig was operated with a controlled Ar atmosphere in the chamber, in order to protect the samples, glassy carbon sample cells and the heating elements from oxidation.

Samples were illuminated with a white X-ray beam peaking sharply at $19 \mathrm{keV}$, produced by an X-ray undulator (U17.6, gap size $20 \mathrm{~mm}$ ). The transmitted beam from the sample was collected onto a $200 \mu \mathrm{m}$ thick LuAG:Ce $\left(\mathrm{Lu}_{3} \mathrm{Al}_{5} \mathrm{O}_{12}\right.$ :Ce) single crystal scintillator, which then projected the image onto a CMOS PCO.Edge 4.2 camera through a $3 \times$ objective lens system. Radiography videos were recorded at $10 \mathrm{~Hz}$ with an image pixel size of $2.2 \mu \mathrm{m} /$ pixel.

Directional solidification from bottom to top was contrived by imposing a thermal gradient across the sample when it was fully liquid and cooling both heaters simultaneously at a constant cooling rate (while maintaining a constant temperature difference between the heaters), through the alloy solidification temperature range. Experiments were performed at two different thermal gradients of $5 \mathrm{~K} \mathrm{~mm}^{-1}$ and $8 \mathrm{~K} \mathrm{~mm}^{-1}$, and four different cooling rates ranging from $0.5 \mathrm{Ks}^{-1}$ to $4 \mathrm{Ks}^{-1}$. Overall, 1,893 IMC particles were studied in 22 experiments (see Table 2). 
Radiographic images acquired from both synchrotron and laboratory apparatus were often affected by sample surface defects and illumination variations due to defects in the optical system. Therefore, the images were processed following a

\section{Results}
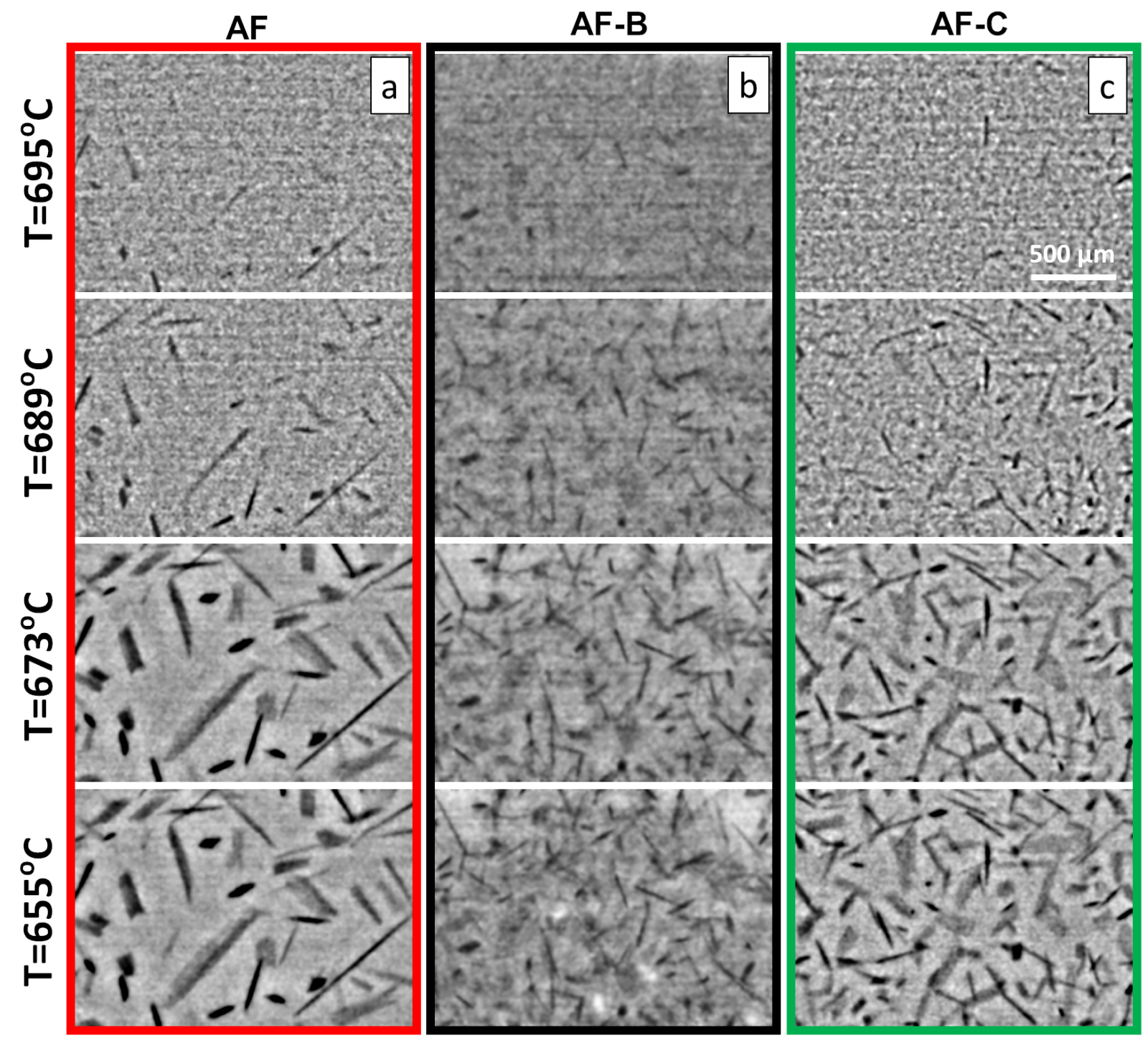

Figure 3: Radiography sequences showing primary IMC formation in the near isothermal, horizontal solidification of alloys (a) AF, (b) AF-B and (c) AF-C, all at a cooling rate of $2 \mathrm{Ks}^{-1}$.

Fig. 3 shows image sequences from the solidification of alloys (a) AF, (b) AF-B and (c) $\mathrm{AF}-\mathrm{C}$, at $2 \mathrm{Ks}^{-1}$ in the horizontal, near isothermal condition. The IMCs 
formed almost uniformly across the field of view, as expected from the absence of a thermal gradient, and without noticeable movement because of the horizontal arrangement. However, due to the relatively poor image quality in the early stages of solidification, where contrast due to Fe segregation was relatively low, the total number of IMC formation events could only be estimated by counting the IMCs at the end of the sequences (rather than frame-by-frame, as they appeared). The final number of IMCs in the field of view was always significantly larger in the inoculated samples: 59 in AF (Fig. 3(a)), 114 in AF-C (Fig. 3(c)) and 161 in AF-B (Fig. $3(b))$.

Fig. 4 shows three image sequences for the alternative vertical, directional solidification again at $2 \mathrm{Ks}^{-1}$ for (a) AF and (b) AF-B at a thermal gradient of $5 \mathrm{~K} \mathrm{~mm}^{-1}$ and (c) AF-B at a thermal gradient of $8 \mathrm{~K} \mathrm{~mm}^{-1}$. The formation of IMCs started near the bottom of the field of view and continued upwards due to the vertical thermal gradient, and solidification finished with the appearance of the eutectic front at $T=655^{\circ} \mathrm{C}$. The number of IMC formation events was counted manually frame-by-frame for each sequence and the total number of IMCs was obtained by summing the number of IMC formation events in each frame. By using this method, the total number of IMCs formed in each solidification sequence was not affected by any disappearance of the IMCs from the field of view due to settling. The total number of IMC formation events (i.e. total number of IMCs formed) in the field of view was 66 in the base alloy (AF), compared with 97 in the $\mathrm{TiB}_{2}$ inoculated sample (AF-B) at $5 \mathrm{~K} \mathrm{~mm}^{-1}$. Upon imposing a higher thermal gradient of $8 \mathrm{~K} \mathrm{~mm}^{-1}$, the total number of IMC formation events in the same AF-B alloy reduced sharply to 26. This trend was consistent across repeated experiments. Figs. 4(d) and (e) show the evolution of the number of IMCs and the formation rate with time, respectively. For each experiment, $t=0$ was set when an IMC first appeared in the field of view, and the formation rate was the highest for AF-B at $5 \mathrm{~K} \mathrm{~mm}^{-1}$.

In contrast to the horizontal condition, there was frequent settling of IMCs in AF-B during vertical solidification because $\mathrm{Al}_{13} \mathrm{Fe}_{4}$ had higher density $\left(3.84 \mathrm{~g} \mathrm{~cm}^{-3}\right)$ than the liquid $\left(2.75 \mathrm{~g} \mathrm{~cm}^{-3}\right)$. The yellow circles in Figs. 4(b) and (c) highlight 


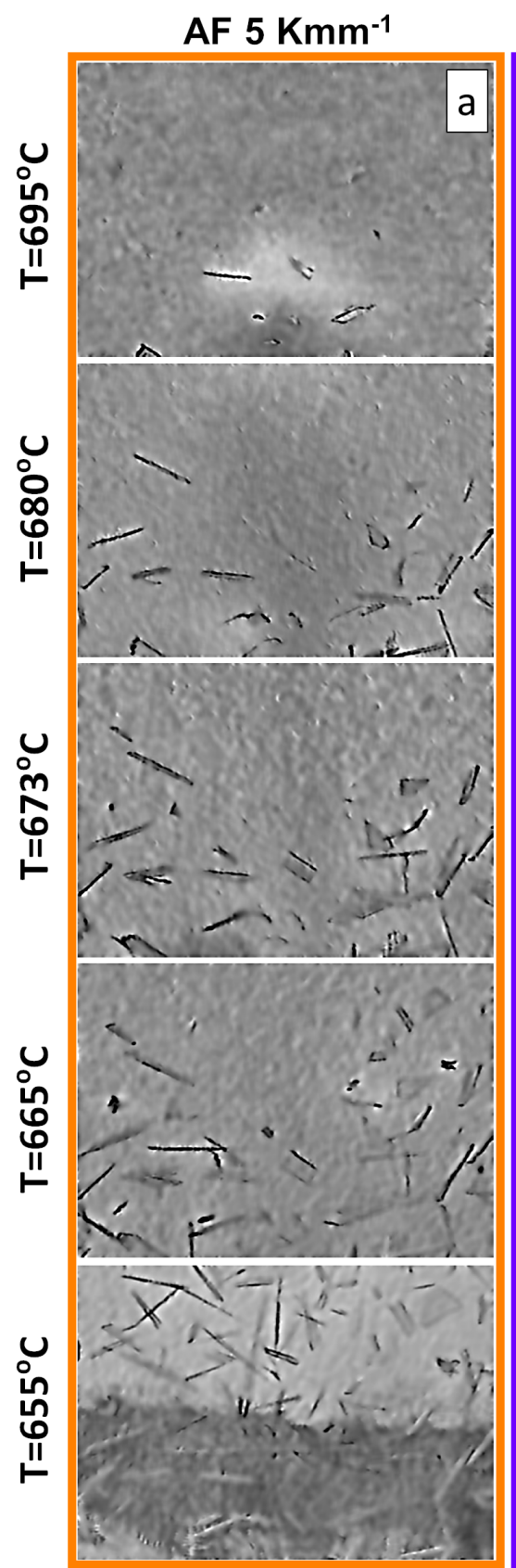

AF-B $5 \mathrm{Kmm}^{-1}$
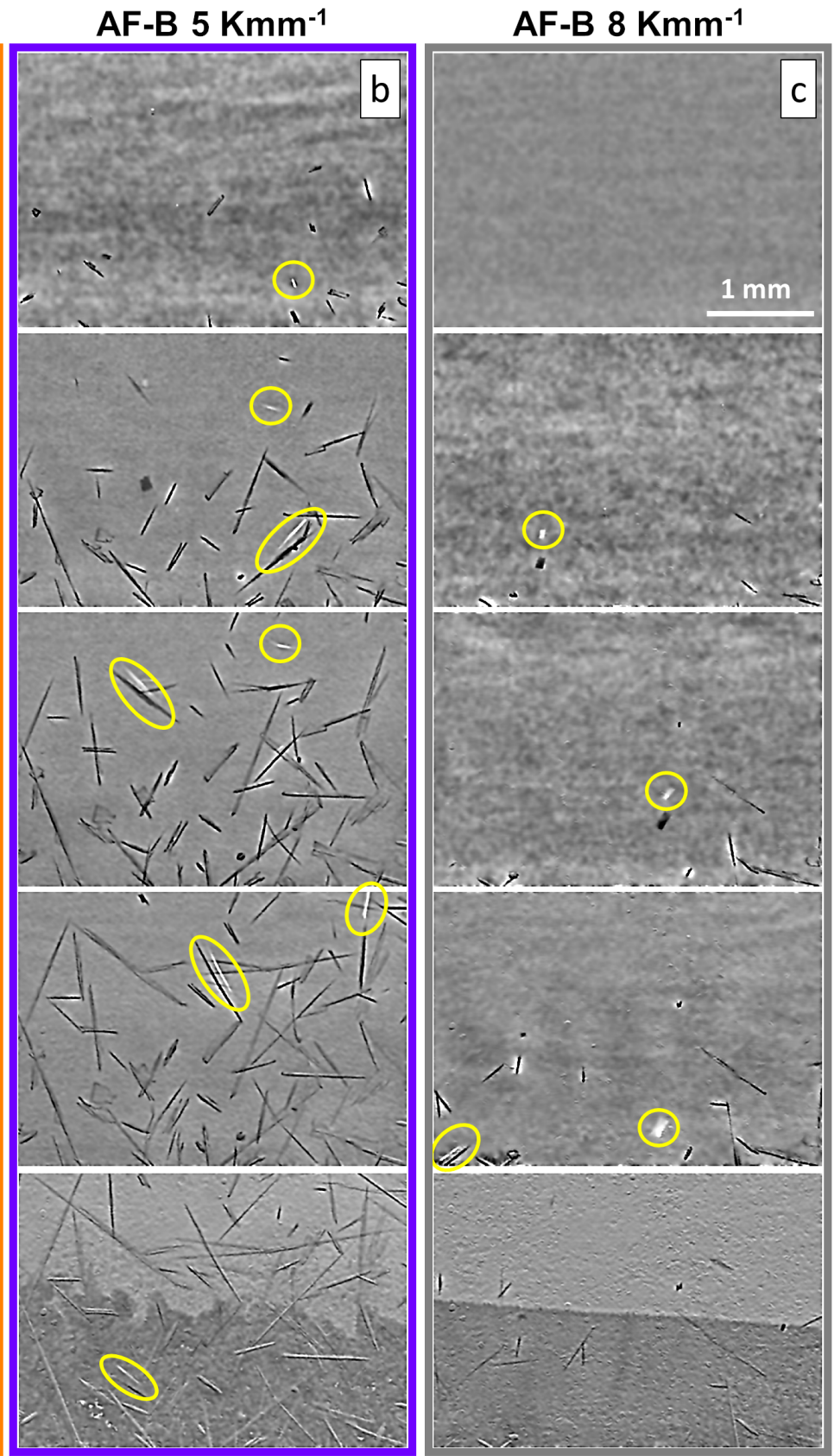

d

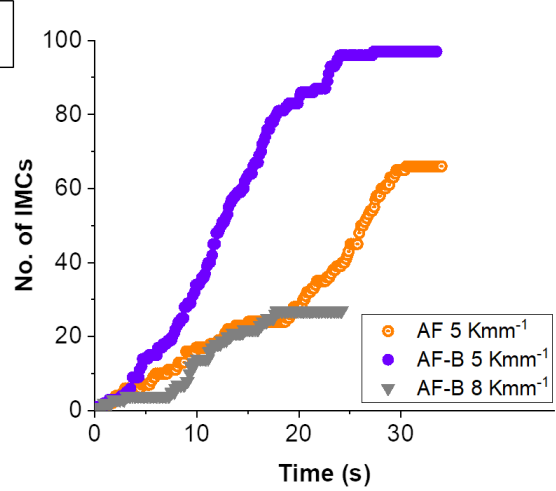

e

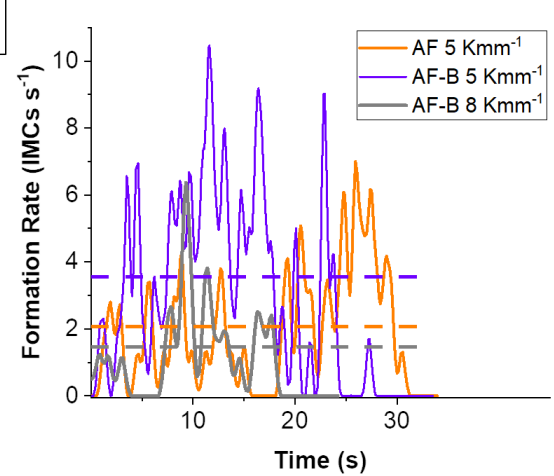

Figure 4: Radiography sequences showing primary IMC formation in directional, vertical solidification at $2 \mathrm{Ks}^{-1}$ of (a) AF and (b) AF-B at a thermal gradient of $5 \mathrm{~K} \mathrm{~mm}^{-1}$, and (c) AF-B at $8 \mathrm{~K} \mathrm{~mm}^{-1}$. Each image was obtained by subtracting frame $f_{n-15}$ from frame $f_{n}$. In AF-B, settling of IMCs was frequently observed, and the yellow circles in (b) and (c) highlight the white "shadow" left behind by the settling IMCs upon image subtraction. (d) Corresponding evolution of the number of IMCs in the field of view, and (e) evolution of formation rate as a function of time. Dashed lines in (e) indicate the average formation rate. For each experiment, $t=0$ was set when the first IMC appeared in the field of view. 
the white "shadow" left behind by settling IMCs due to the image subtraction algorithm used to improve image quality (see S1.1 in the Supplementary Materials). In contrast, all the IMCs remained static after formation in alloy $\mathrm{AF}$, without inoculation. This is discussed in more detail in the next section.

Figs. 5(a) to (c) show radiographs towards the end of solidification at different cooling rates for AF-B under near isothermal, horizontal conditions. The number of IMCs formed in the field of view increased significantly with increasing cooling rate, from 31 at $0.5 \mathrm{Ks}^{-1}$ to 85 at $1 \mathrm{Ks}^{-1}$ and further to 161 at $2 \mathrm{Ks}^{-1}$. However, when a thermal gradient of $5 \mathrm{~K} \mathrm{~mm}^{-1}$ was applied to the same alloy, as shown in Figs. 5(d) to (f), the number of IMCs had a very weak sensitivity to cooling rate: 94 at $0.5 \mathrm{Ks}^{-1}, 97$ at $2 \mathrm{Ks}^{-1}$ and 92 at $4 \mathrm{Ks}^{-1}$.
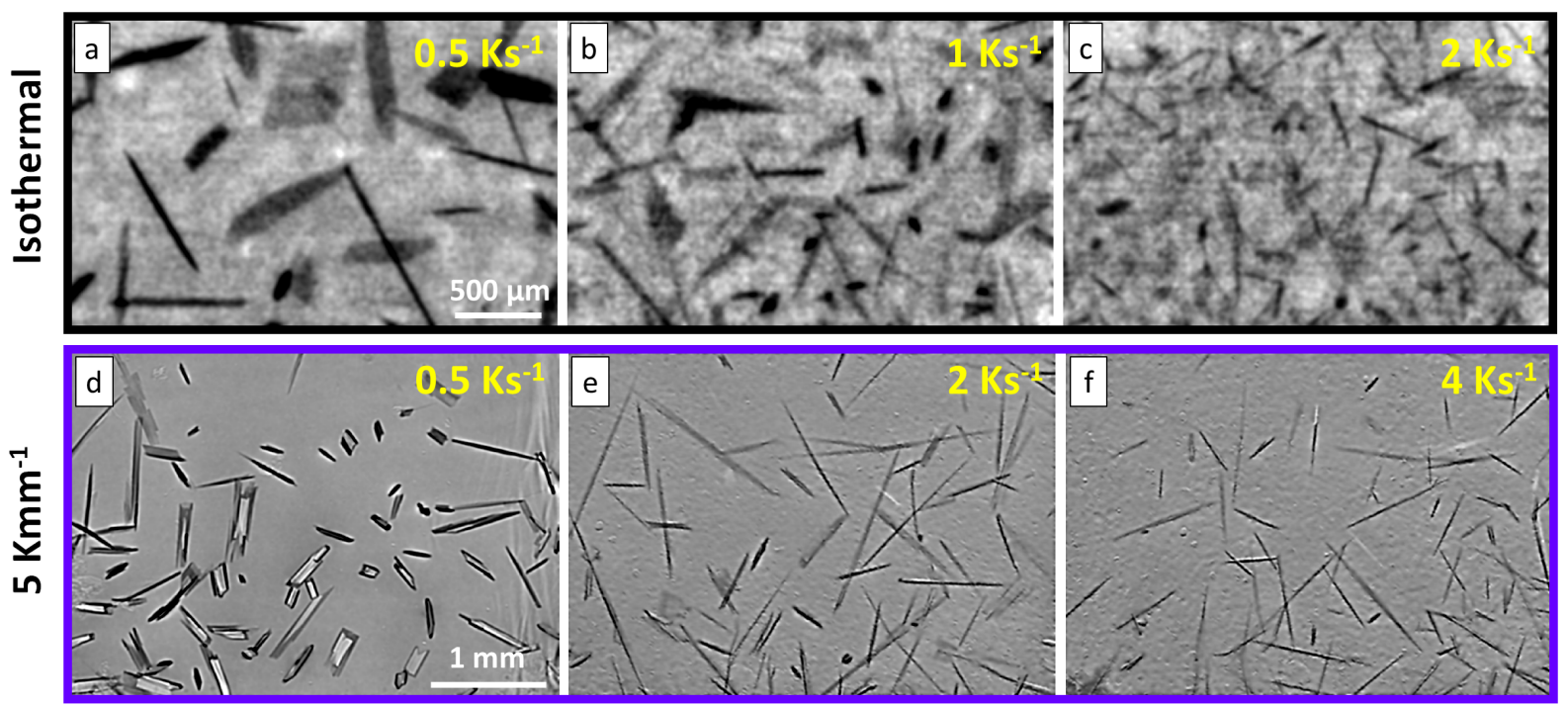

Figure 5: Typical radiographs towards the end of solidification at (a) $0.5 \mathrm{Ks}^{-1}$ (b) $1 \mathrm{Ks}^{-1}$ and (c) 2 $\mathrm{Ks}^{-1}$ for AF-B, under near isothermal, horizontal conditions. Typical radiographs towards the end of solidification at (d) $0.5 \mathrm{Ks}^{-1}$ (e) $2 \mathrm{Ks}^{-1}$ and (f) $4 \mathrm{Ks}^{-1}$ for AF-B at $5 \mathrm{~K} \mathrm{~mm}^{-1}$. Note that the field of view in (a) to (c) is approximately 2.5 times smaller than (d) to (f).

By dividing the total number of IMCs by the volume of the field of view in each experiment, Fig. 6(a) summarises the total IMC density in alloys AF, AF-B and AF-C as a function of cooling rate for 34 experiments in the near isothermal, horizontal arrangement. AF-B and AF-C both showed a higher IMC density than AF, particularly at higher cooling rates, whilst the AF-B alloy had the highest IMC density over the range of cooling rates. Dividing the IMC density by the duration 

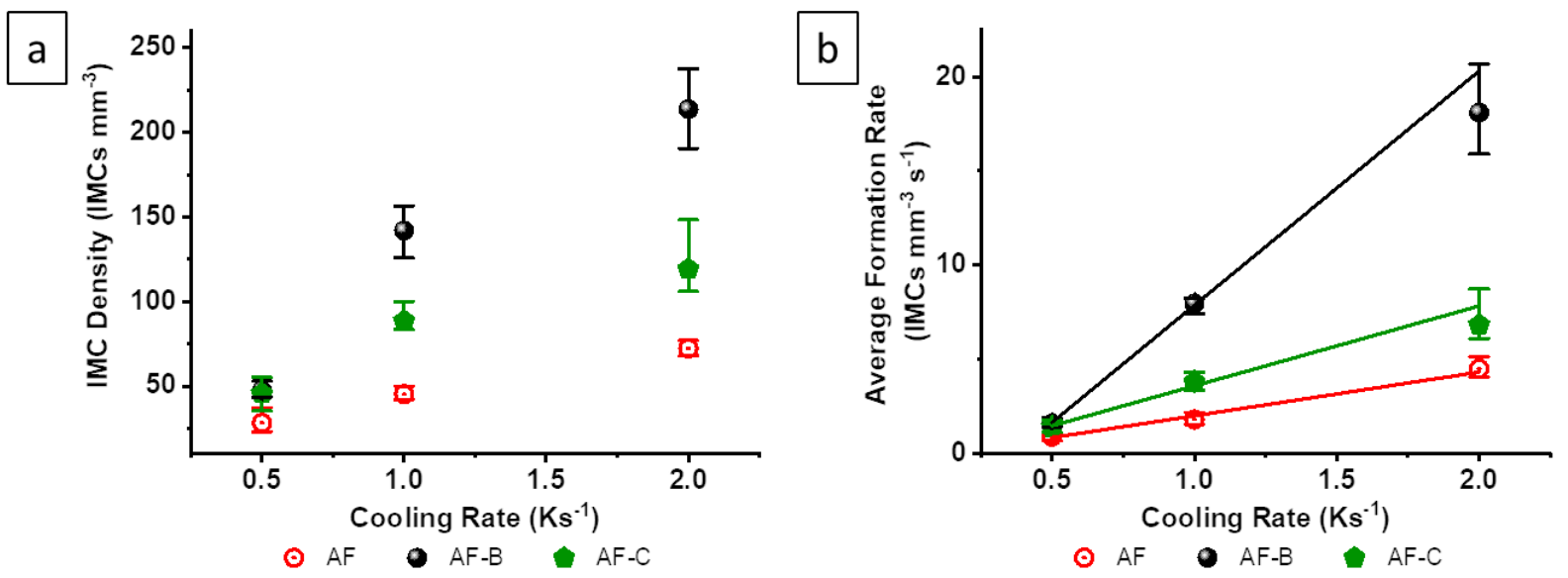

Figure 6: (a) Total IMC density (total number of IMCs divided by the volume of the field of view) and (b) average formation rate (total IMC density divided by the duration of IMC formation) in alloys $\mathrm{AF}, \mathrm{AF}-\mathrm{B}$ and AF-C at $0.5-2 \mathrm{Ks}^{-1}$ in near isothermal, horizontal solidification. The solid lines in (b) indicate a linear best-fit to highlight the trend.

of IMC formation (i.e. time between the first and last IMC appearing in the field of view), the average IMC formation rate in $\mathrm{AF}, \mathrm{AF}-\mathrm{B}$ and $\mathrm{AF}-\mathrm{C}$ increased steadily with cooling rate (Fig. 6(b)), and AF-B showed the greatest sensitivity to cooling rate.

Figs. [7(a) and (b) summarise the total IMC density (total number of timeresolved formation events divided the volume of the field of view) and corresponding average formation rate in 22 directional, vertical solidification experiments of AF and $\mathrm{AF}-\mathrm{B}$ respectively. IMC density and average formation rate for $\mathrm{AF}-\mathrm{B}$ were consistently higher than for $\mathrm{AF}$ at the same thermal gradient of $5 \mathrm{~K} \mathrm{~mm}^{-1}$. However in comparison with the near isothermal counterparts, solidification with a thermal gradient showed much weaker IMC density sensitivity to cooling rate (Fig. 7(a)), especially for inoculated alloys (typical of Figs. 5(d) to (f)). Nonetheless, there was again a strong relationship between the average IMC formation rate and cooling rate (Fig. 7(b)). At an increased thermal gradient of $8 \mathrm{~K} \mathrm{~mm}^{-1}$, the IMC density

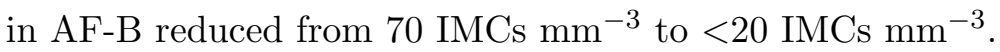

To further illustrate the effects of thermal gradient, Fig. 8 summarises the IMC density and average formation rate data for near isothermal, $5 \mathrm{~K} \mathrm{~mm}^{-1}$ and $8 \mathrm{~K} \mathrm{~mm}^{-1}$ for AF-B. Increasing thermal gradient generally reduced both the IMC density and average formation rate, and the effect was more pronounced at higher 


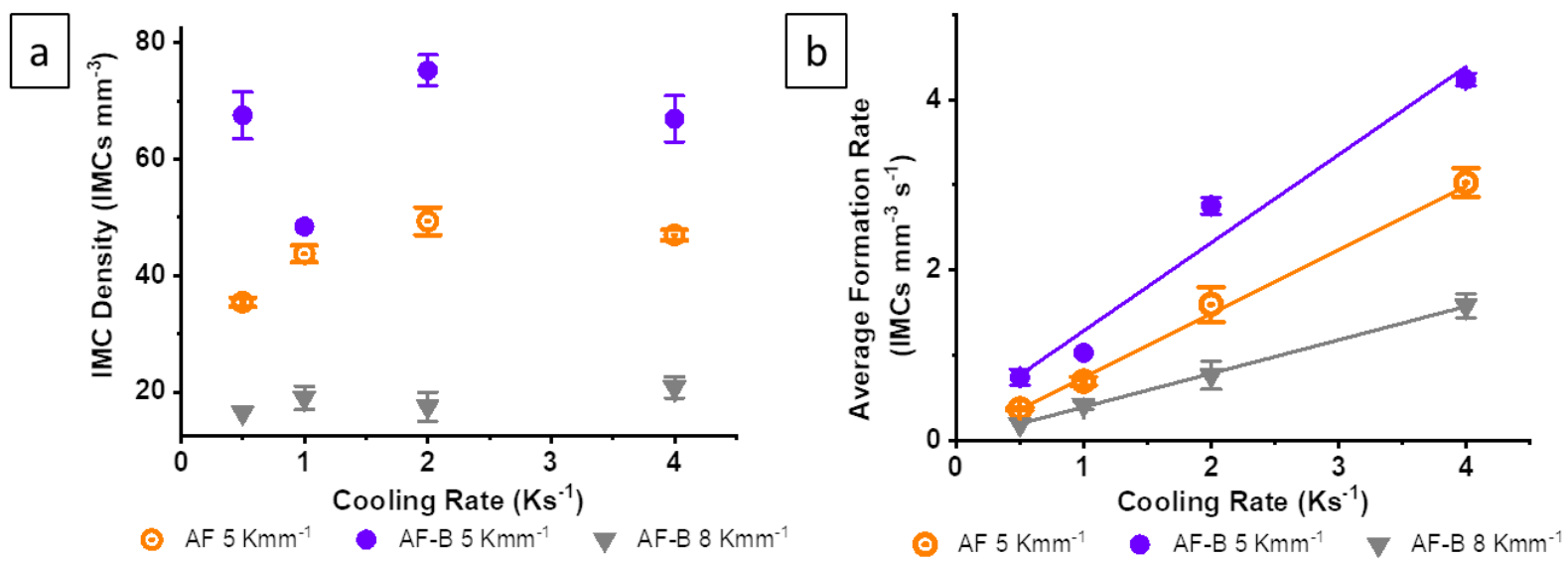

Figure 7: (a) Total IMC density (total number of time-resolved IMC formation events divided by the volume of the field of view) and (b) average formation rate in AF and AF-B at a thermal gradient of $5 \mathrm{~K} \mathrm{~mm}^{-1}$ and AF-B at a thermal gradient of $8 \mathrm{~K} \mathrm{~mm}^{-1}$, at cooling rates of 0.5 to $4 \mathrm{Ks}^{-1}$ in directional, bottom-to-top solidification. The solid lines in (b) indicate a linear best-fit to highlight the trend.

cooling rate: at $2 \mathrm{Ks}^{-1}$, the IMC density decreased from $214 \pm 24 \mathrm{IMCs} \mathrm{mm}^{-3}$ at near isothermal condition to $17 \pm 3$ IMCs $\mathrm{mm}^{-3}$ at the highest thermal gradient of $8 \mathrm{~K} \mathrm{~mm}^{-1}$. The corresponding average formation rate was also significantly reduced

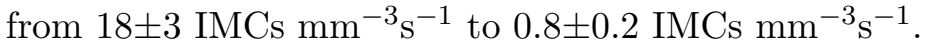
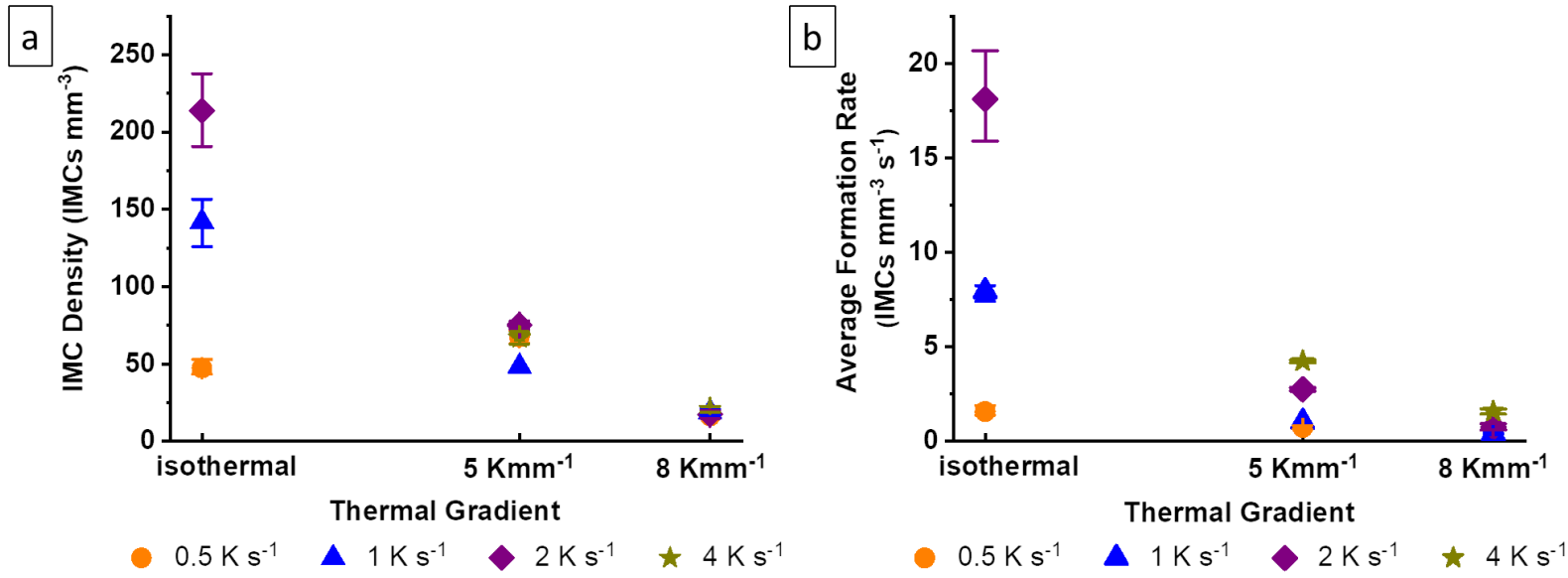

Figure 8: (a) IMC density as a function of thermal gradient for alloy AF-B. (b) Average IMC formation rate as a function of thermal gradient for alloy AF-B. 


\section{Discussion}

\subsection{IMC nucleants}

In all conditions, inoculant additions increased the number of IMC formation events (Figs. 6 and 7), suggesting that $\mathrm{TiB}_{2}$ and $\mathrm{TiC}$ catalysed the nucleation of IMCs. As pointed out previously in Figs. 4(b) and (c), when AF-B was solidified vertically, there was frequent settling of IMCs. By comparison, in AF all the IMCs remained static once formed, and this is interpreted as the IMCs primarily nucleating on the interior side of the oxide skin of the sample. In contrast, frequent IMC settling in AF-B suggested that $\mathrm{TiB}_{2}$ particles suspended in the melt catalysed nucleation of IMCs. Therefore, settling of the IMCs helped distinguish IMCs nucleating on $\mathrm{TiB}_{2}$ particles (that were largely mobile) and IMCs mostly likely nucleating on the oxide skin (that were largely immobile).

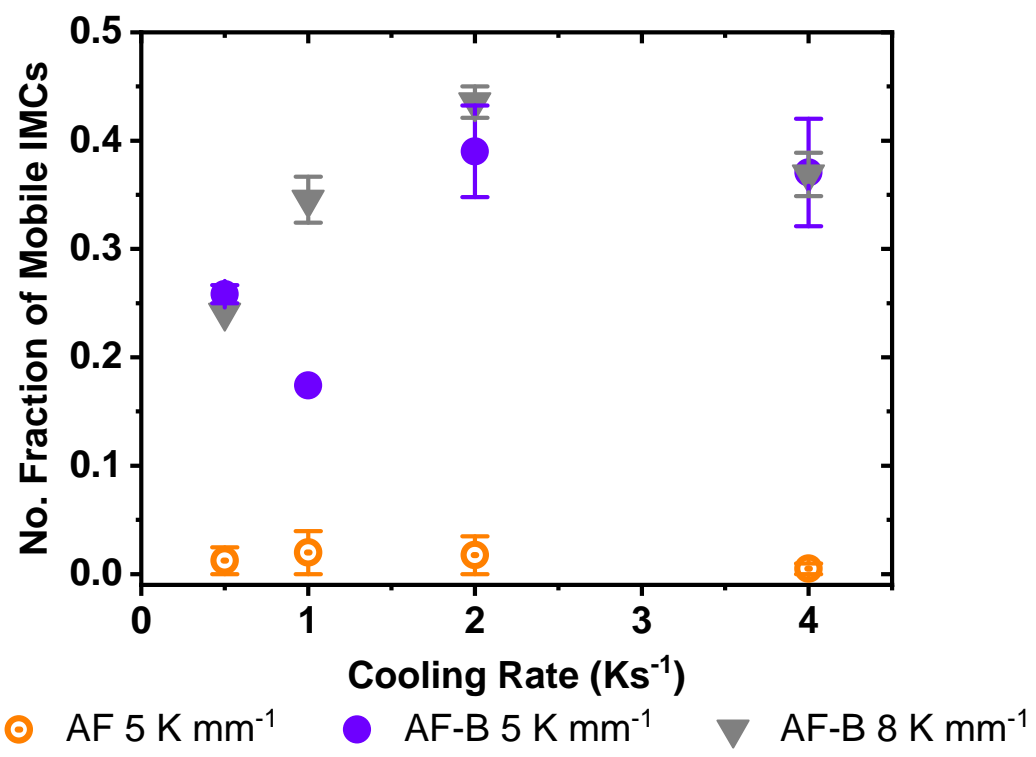

Figure 9: Number fraction of mobile primary IMCs as a function of cooling rate for AF and AF-B at $5 \mathrm{~K} \mathrm{~mm}^{-1}$ and AF-B at $8 \mathrm{~K} \mathrm{~mm}^{-1}$.

In either $\mathrm{AF}$ or $\mathrm{AF}-\mathrm{B}$, the number fraction of mobile IMCs shown in Fig. 9 had no obvious relationship with cooling rate. In AF nearly all IMCs remained static, because they nucleated on the oxide wall of the sample. This finding is consistent with previous observations by post-solidification microscopy [25] 27, 43]. 
For example, Cao and Campbell investigated the role of oxide films on the formation of the Fe-rich IMCs in Al-Si alloys with various Fe concentrations [25, 26]. There were oxide bifilms entrained in the melt with two surfaces: a dry inner surface and a wetted exterior surface that acted as preferred substrate for the nucleation and growth of Fe-rich IMCs [25, 26].

The minor fraction of mobile IMCs $(\sim 2 \%)$ in $\mathrm{AF}$ could be a consequence of nucleation on minor inclusions in the liquid, or detachment from the oxide wall due to convection in the liquid. In AF-B, up to $45 \%$ of the primary IMCs were mobile regardless of thermal gradient or cooling rate, suggesting that they nucleated on $\mathrm{TiB}_{2}$ particles. This provides evidence that $\mathrm{TiB}_{2}$ can nucleate Fe-rich IMCs (at least as primary phase), as suggested by earlier researchers based on extraction and microscopy [29, 44, 45].

\subsection{IMC formation}

The IMC density and average formation rate decreased as the thermal gradient increased, from a near isothermal condition to $5 \mathrm{~K} \mathrm{~mm}^{-1}$, and then further to $8 \mathrm{~K} \mathrm{~mm}^{-1}$ (Fig. 8). Reduced grain formation events with increasing thermal gradient was also reported by $\mathrm{Xu}$ et al. [42] for equiaxed $\alpha$ - $\mathrm{Al}$ dendrites in an $\mathrm{Al}-20 \mathrm{Cu}$ (wt\%) alloy for near isothermal, $5 \mathrm{~K} \mathrm{~mm}^{-1}$ and $10 \mathrm{~K} \mathrm{~mm}^{-1}$ conditions.

The IMC formation behaviour under near isothermal and directional solidification can be explained by considering the undercooling conditions experienced by nucleants during cooling. Within the melt, potential nucleants are assumed to be more or less uniformly distributed, and according to the free growth model [46] they have a distribution of efficiencies related to the distribution of diameters. It follows that the larger the undercooled zone, the greater the probability that it contains an efficient nucleant particle, and larger undercoolings have a greater probability of activating a nucleant and forming a new IMC. 


\subsubsection{The undercooled zone (UZ) in front of a growing IMC}

The size of the $U Z$ and the magnitude of undercooling experienced by a potential nucleant for an IMC in the $U Z$ relates to the distribution of temperature and solute in the liquid [47. Solute fields are particularly important in the vicinity of preexisting IMCs, whereas the liquid solute field is generally flat (well-mixed) far from regions of prior solidification (as shown experimentally later).

To investigate the solute fields, 60 solute profiles around 7 IMCs were measured from a solidification sequence using synchrotron X-ray radiography at $0.5 \mathrm{Ks}^{-1}$ and $5 \mathrm{~K} \mathrm{~mm}^{-1}$. The image quality of this sequence was sufficiently high as to be able to extract solute concentration from pixel intensities. More detail of the method to calculate solute field from X-ray intensities can be found in S1.2 in the Supplementary Materials (and in [48, 49]). A video of the evolution of solute concentration in the liquid is also available in the Supplementary Materials.

Fig. 10(a) shows the solute measurement approach in more detail using a radiograph at an early stage of solidification when IMCs were well-separated, Fig. 10(b) shows a map of the solute field around IMC A, visualised as a polar image centred on A, and Fig. 10(c) is a plot of the measured Fe concentration along the radial direction as indicated by the red arrow in Figs. 10(a) and (b) $\left(\Theta=18^{\circ}\right)$.

The solute concentration $C_{l}$ in the liquid in front of a growing phase in a binary alloy follows [50, 51]:

$$
C_{l}=C_{0}+\Delta C_{0} e^{-x \frac{v}{D_{l}}}
$$

where $C_{0}$ is the bulk liquid composition, $\Delta C_{0}$ is the composition difference between the bulk liquid and the liquid at the interface, $D_{l}$ is the solute diffusion coefficient in the liquid, $v$ is the interface velocity, and $x$ is the distance from the interface. The pre-exponential term $\Delta C_{0}$ is defined as $C_{0} \frac{1-k}{k}$. For hypereutectic Al-Fe alloys where the partition coefficient is $k>1, \Delta C_{0}$ is negative meaning that the solute segregates preferentially into the IMC, depleting the liquid ahead of the interface, as shown experimentally in Fig. 10(c), and the liquid concentration is the nominal 
alloy composition of $3 \mathrm{wt} \%$ Fe further than $\sim 1 \mathrm{~mm}$ from the solid/liquid interface.
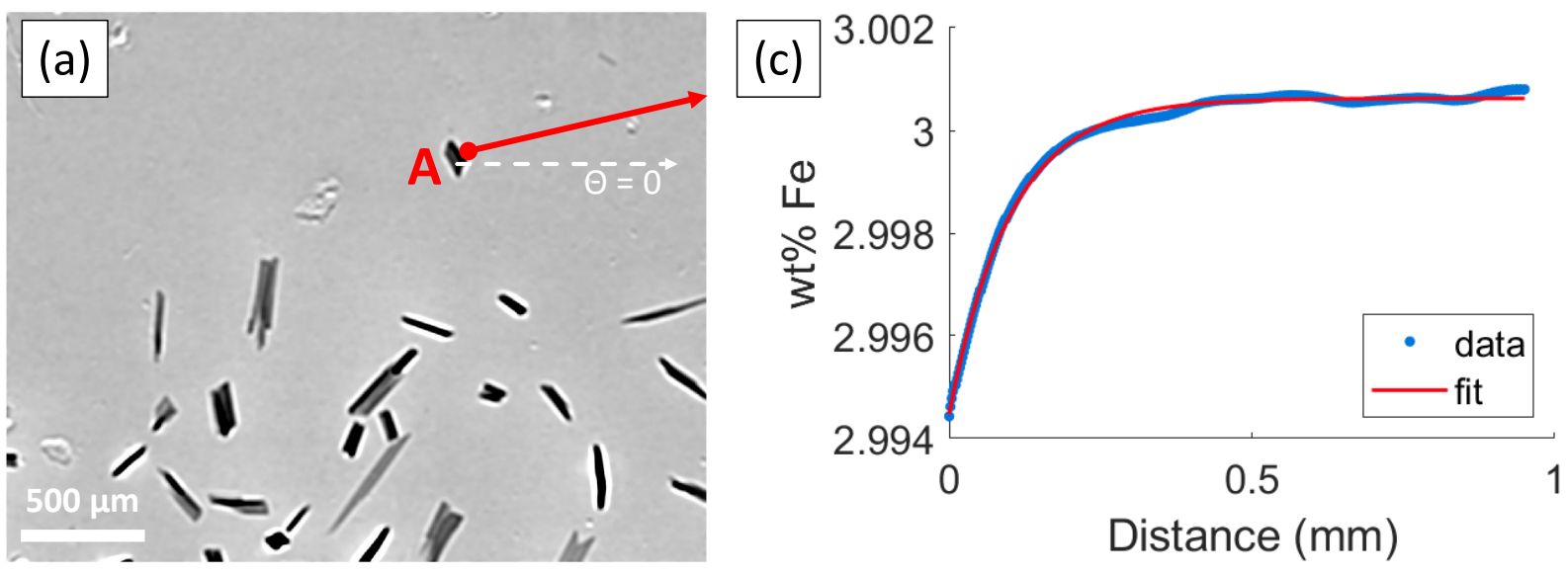

\section{(b)}

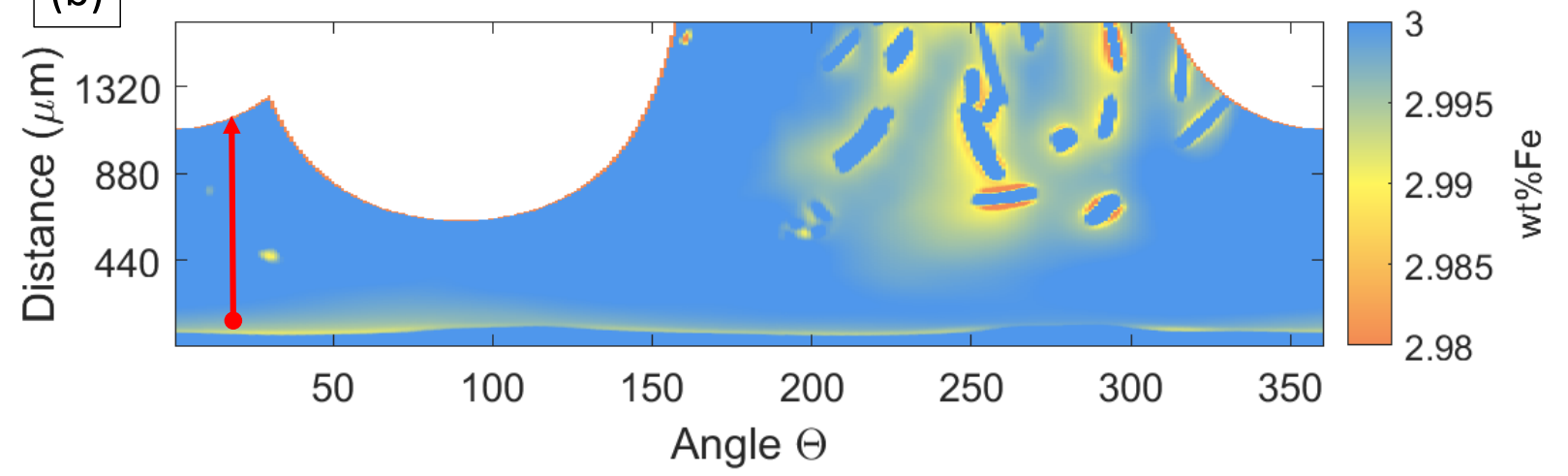

Figure 10: (a) A radiograph ( $t=37 \mathrm{~s}$ ) of AF-B solidifying at $0.5 \mathrm{Ks}^{-1}$ and $5 \mathrm{~K} \mathrm{~mm}^{-1}$. (b) The solute field around IMC A visualised as a polar image centred at A. $\Theta$ increases anticlockwise. The y-axis is the radial distance from the IMC centre. (c) The solute profile with a best-fit $\left(R^{2}=0.996\right)$ to Eq. 1 along the direction indicated by the red arrow in (a) and (b).

The $C_{l}$ profile was fitted to Eq. 1 as shown in Fig. 10(c), with $R^{2}=0.996$, indicating an excellent fit to Eq. 1. Assuming $D_{l}=2.82 \times 10^{-9} \mathrm{~m}^{2} \mathrm{~s}^{-1}$ [52], the bestfit parameters were: $C_{0}=3.0007 \mathrm{wt} \%, \Delta C_{0}=-0.0061 \mathrm{wt} \%, v=28 \mu \mathrm{ms}^{-1}$ and $\delta_{c}=$ $204 \mu \mathrm{m} . \delta_{c}=2 \frac{D_{l}}{v}$ is the equivalent boundary layer, which is a measure of the extent of the solute field. The averages for all 60 solute profiles were: $\bar{R}^{2}=0.940 \pm 0.062$, $\bar{C}_{0}=3.0004 \pm 0.0006 \mathrm{wt} \%, \overline{\Delta C}_{0}=-0.0053 \pm 0.0023 \mathrm{wt} \%, \bar{v}=33 \pm 25 \mu \mathrm{ms}^{-1}$ and $\bar{\delta}_{c}=244 \pm 148 \mu \mathrm{m} . \bar{C}_{0}$ agreed with the nominal alloy composition, while $\Delta C_{0}$ was significantly smaller than might be expected because of the quasi-3D experimental set-up i.e. IMCs are only a few microns in thickness but sit in a $200 \mu \mathrm{m}$ thick sample volume. Because measured concentrations at each pixel were an average of the Fe concentration through the sample thickness, the measured solute concentration 
close to the solid/liquid interface will inevitably be higher than actual due to the relatively more concentrated surrounding liquid. IMC growth velocities in the same solidification sequence were also measured directly by hand from the radiographs [36], and were $27 \pm 7 \mathrm{mms}^{-1}$ for non-constrained IMCs (i.e. IMCs growing with no physical or solute field impingement) and $7 \pm 3 \mu \mathrm{ms}^{-1}$ for constrained IMCs. The best-fit velocities to Eq. 1 (based on the solute profile only) were thus in good agreement with those measured directly from the videos.

From Eq. 1, the equilibrium liquidus temperature $T_{l}$ in the solute field ahead of a growing IMC is:

$$
T_{l}=T_{0}+m \Delta C_{0} e^{-x \frac{v}{D_{l}}}
$$

where $T_{0}$ is the liquidus temperature at composition $C_{0}$, and $m$ is the slope of the liquidus. The temperature field $T_{q}$ at a thermal gradient $G$ imposed in the $x$-direction is:

$$
T_{q}=T_{l(x=0)}+x G=T_{0}+m \Delta C_{0}+x G
$$

Fig. 11(a) is a 1-D schematic representation of the liquid ahead of a growing IMC under the three conditions studied in this work. The undercooled regions are shaded for the isothermal condition and two thermal gradient conditions $(G 1<G 2)$. From Eqs. 2 and 3 , the magnitude of undercooling $\Delta T$ in the shaded region is given by:

$$
\begin{aligned}
\Delta T(x, G, v) & =T_{l}-T_{q} \\
& =T_{0}+m \Delta C_{0} e^{-x \frac{v}{D_{l}}}-\left(T_{0}+m \Delta C_{0}+x G\right) \\
& =m \Delta C_{0}\left(e^{-x \frac{v}{D_{l}}}-1\right)-x G
\end{aligned}
$$

Assuming no interactions between variables, the resulting form of $\Delta T(x, G, v)$ is shown schematically in Fig. 11(b). It is helpful to clarify that Eq. 4 describes the 


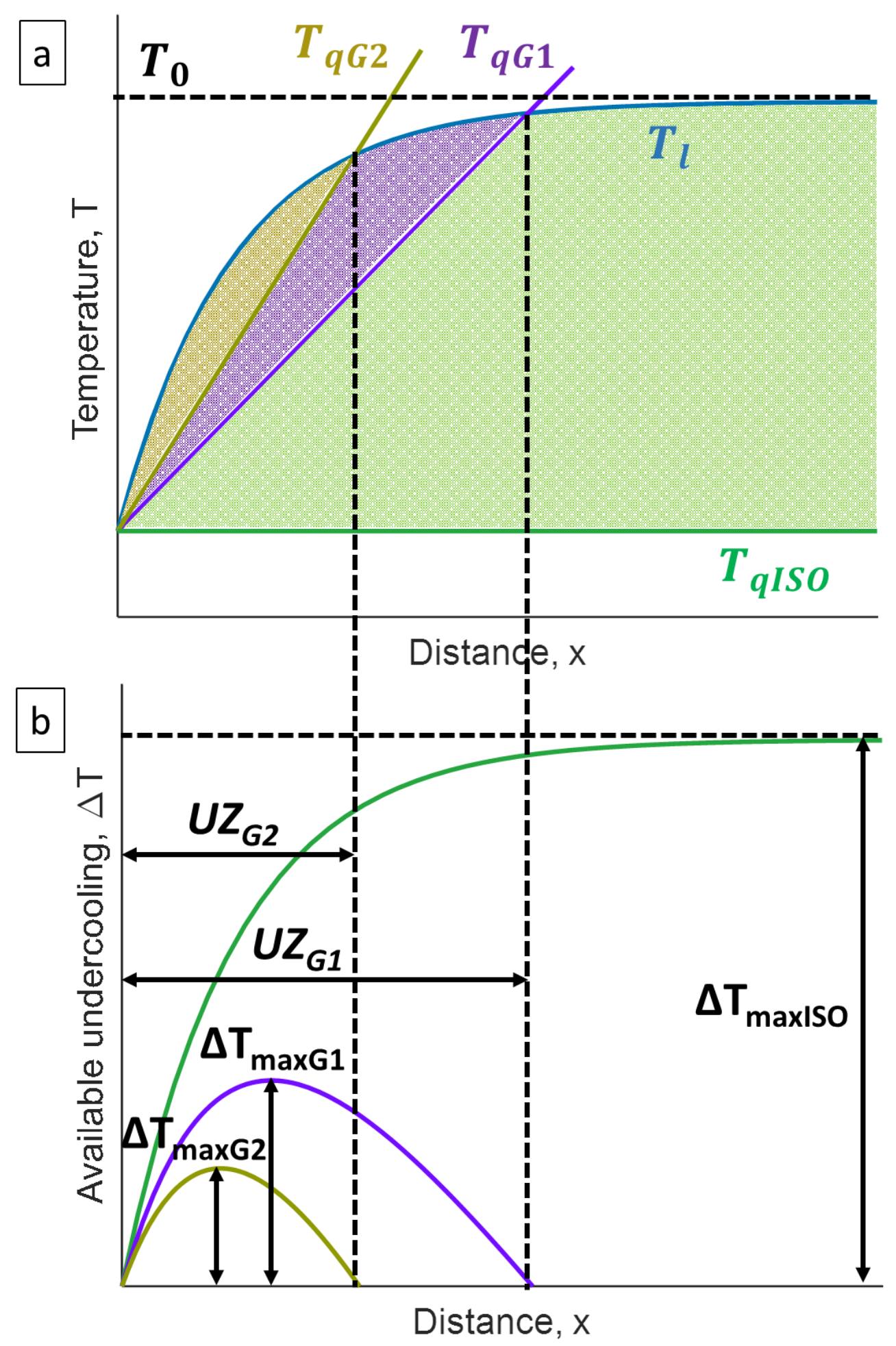

Figure 11: (a) A schematic of the undercooled zone $(U Z)$ in front of a growing IMC in the isothermal condition, and with thermal gradients of $G 1$ and $G 2(G 2>G 1) . T_{l}$ is the equilibrium liquidus temperature, and $T_{0}$ is the bulk liquidus. $T_{q I S O}, T_{q G 1}$ and $T_{q G 2}$ are the imposed temperature profiles under the isothermal condition, $G 1$ and $G 2$ respectively. (b) Undercooling curves at the isothermal condition, $G 1$ and $G 2 . U Z_{G 1}$ and $U Z_{G 2}$ denote the size of $U Z$ at $G 1$ and $G 2$, respectively. $\Delta T_{\max I S O}, \Delta T_{\max G 1}$ and $\Delta T_{\max G 2}$ are the maximum available undercooling at the isothermal condition, $G 1$ and $G 2$ respectively. 
available undercooling, not necessarily the actual undercooling that might be measured in an experiment for an individual IMC formation event, which is different and better termed the nucleation undercooling. Under idealised isothermal conditions, $U Z$ extends across the entire volume of the melt. Under a thermal gradient, the $U Z$ is only adjacent to the growing IMC. As the thermal gradient is increased, the $U Z$ width is reduced from $U Z_{G 1}$ to $U Z_{G 2}$ as shown in Figs. 11(a) and (b).

The size of the undercooled zone $U Z(G, v)$ is given by imposing the condition $\Delta T=0$ in Eq. 4 and solving for $x$ :

$$
U Z(G, v)=-\frac{1}{G}\left[\Delta C_{0} m-\frac{D_{l}}{v} G W_{0}\left(\frac{\Delta C_{0} m}{G} \frac{v}{D_{l}} e^{\frac{\Delta C_{0} m}{G} \frac{v}{D_{l}}}\right)\right]
$$

where $W_{0}$ is the principal branch of the Lambert $\mathrm{W}$ function [53]. A further derivation of Eq. 5 can be found in S1.3 in the Supplementary Materials.

The maximum undercooling $\Delta T_{\max }(G, v)$ can be obtained by setting the first derivative of Eq. 4 to zero and yields:

$$
\Delta T_{\max }(G, v)=G \frac{D_{l}}{v}\left[\ln \left(-\frac{G}{m \Delta C_{0}} \frac{D_{l}}{v}\right)-1\right]-m \Delta C_{0}
$$

For this hypereutectic Al-Fe alloy, the term $-m \Delta C_{0}$ is positive. As already shown schematically in Fig. 11, the largest $\Delta T_{\max }$ is obtained at $G \rightarrow 0$ (isothermal) as $\lim _{G \rightarrow 0} G \frac{D_{l}}{v}\left[\ln \left(-\frac{G}{m \Delta C_{0}} \frac{D_{l}}{v}\right)-1\right]=0$, and is:

$$
\Delta T_{\max I S O}=-m \Delta C_{o}
$$

Eqs. 5 and 6 show that for a given alloy composition the volume of the liquid undercooled and the magnitude of the undercooling are functions of the imposed thermal gradient and the growth velocity, which in turn is related to cooling rate. The following sections use these expressions to rationalise the experimental effect of increasing the thermal gradient and cooling rate on IMC formation. 


\subsubsection{Effect of thermal gradient}

Figs. 12 (a) and (b) show plots of $U Z$ and $\Delta T_{\max }$ as a function of thermal gradient from Eqs. 5 and 6 respectively for a range of $v$ representative of the experiments. Fig. 12(a) shows that increasing the thermal gradient had a strong non-linear effect

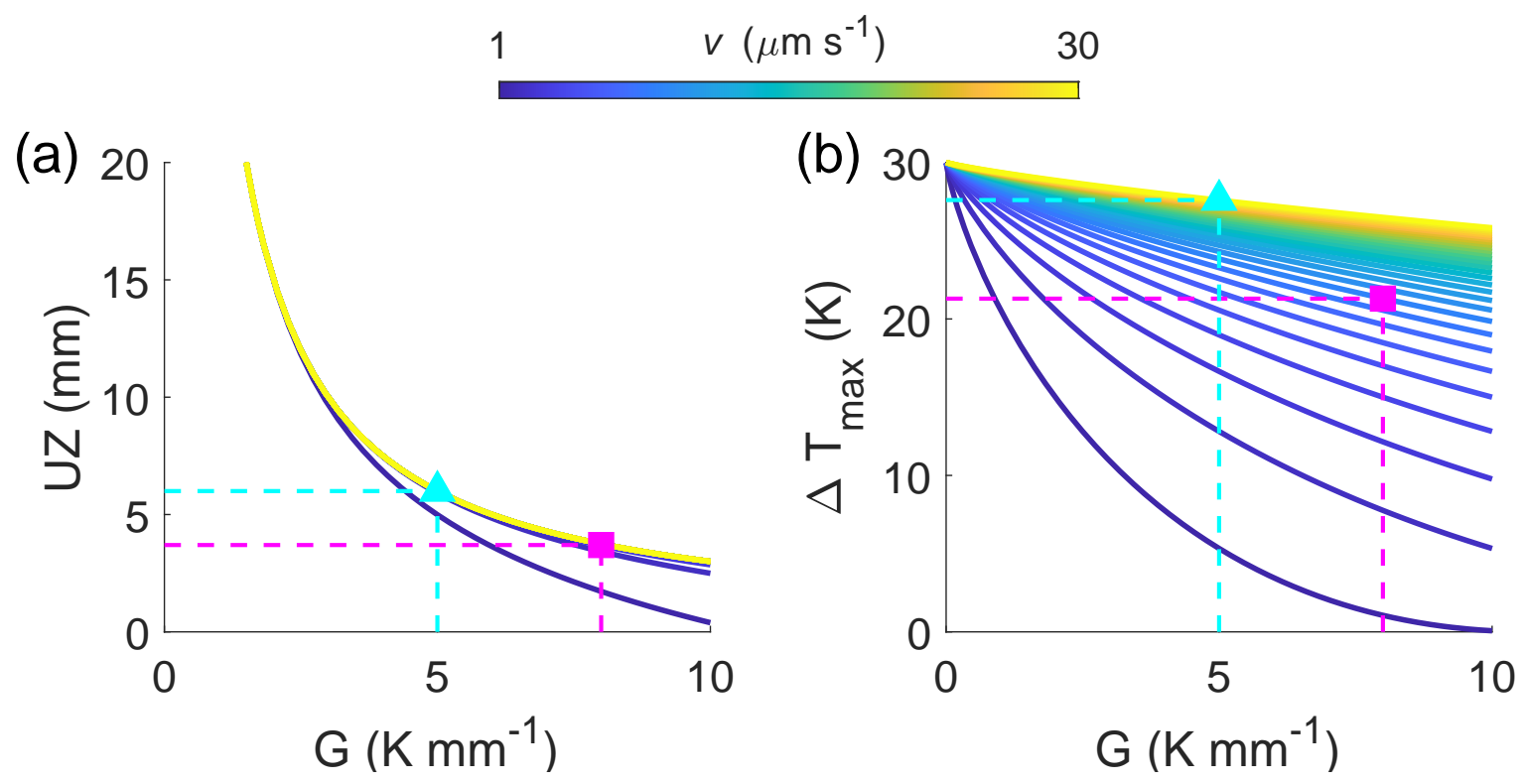
on reducing $U Z$. For example, at a growth velocity of $30 \mu \mathrm{ms}^{-1}, U Z$ decreased from $\sim 15 \mathrm{~mm}$ at $G=2 \mathrm{~K} \mathrm{~mm}^{-1}$ to $6 \mathrm{~mm}$ at $G=5 \mathrm{~K} \mathrm{~mm}^{-1}$ and further to 3.7 $\mathrm{mm}$ at $G=8 \mathrm{~K} \mathrm{~mm}^{-1}$. The growth velocity $v$ had a comparatively weak effect on $U Z$, and was only influential at low growth velocities $<10 \mu \mathrm{ms}^{-1}$. Note that as previously described, $\Delta C_{0}$ was not available from experiment and a value of $\Delta C_{0}=-1 \mathrm{wt} \%$ was assumed, which was consistent with previous observations of the non-equilibrium concentration profiles of Fe-rich IMCs.

Figure 12: (a) $U Z$ and (b) $\Delta T_{\max }$ calculated from Eqs. 5 and 6 as a function of the thermal gradient $G$ and for growth velocities $v$ between $1 \mu \mathrm{ms}^{-1}$ and $30 \mathrm{\mu ms}^{-1}$. The following parameters were used: $\Delta C_{0}=-1 \mathrm{wt} \%, D_{l}=2.82 \times 10^{-9} \mathrm{~m}^{2} \mathrm{~s}^{-1}, m=30 \mathrm{Kwt} \%^{-1}$. The triangles in cyan in (a) and (b) denote $5 \mathrm{~K} \mathrm{~mm}^{-1}$ and a typical growth velocity of $30 \mathrm{\mu m} \mathrm{s}^{-1}$, and the squares in magenta denote $8 \mathrm{~K} \mathrm{~mm}^{-1}$ and a typical growth velocity of $10 \mu \mathrm{m} \mathrm{s}^{-1}$.

$\Delta T_{\max }$ was similarly reduced by increasing $G$, especially at low growth velocities. It should be noted that there is an inter-dependency of growth velocity on the thermal gradient. At a cooling rate of $0.5 \mathrm{Ks}^{-1}$, unconstrained (isolated) IMCs had an average growth velocity of $27 \pm 7 \mu \mathrm{m} \mathrm{s}^{-1}$ at $5 \mathrm{~K} \mathrm{~mm}^{-1}$, which reduced to $9 \pm 3$ $\mu \mathrm{m} \mathrm{s}^{-1}$ when the thermal gradient increased to $8 \mathrm{~K} \mathrm{~mm}^{-1}$. These two points from 
experiment are superimposed on the plots, showing the corresponding estimated values of $U Z$ and $\Delta T_{\max } . \Delta T_{\max }$ estimates of $20-30 \mathrm{~K}$ in Fig. 12(b) are the largest available undercooling. In practice, nucleation likely occurs at a lower undercooling, and consistent with an undercooling of $15 \mathrm{~K}$ at $0.7 \mathrm{Ks}^{-1}$ measured by calorimetry [54]. $U Z$ estimates of several millimetres are in good agreement with analysis for a solidifying Al-7Si (wt\%) alloy under a thermal gradient [47].

\subsubsection{Effect of cooling rate}

In the near isothermal condition, increasing the cooling rate led to a higher IMC density (Fig. 8(a)), but this effect was much less pronounced when a thermal gradient was applied, and then increased.

Fig. 13(a) shows again schematically the liquidus temperature $T_{l}$ ahead of a growing IMC at two cooling rates, $\dot{T}_{1}$ and $\dot{T}_{2}\left(\dot{T}_{1}<\dot{T}_{2}\right)$ according to Eq. 2 At a higher imposed cooling rate, the growth velocity $v$ increased [36], which gives rise to a steeper liquidus profile according to Eq. 2, i.e less diffusion to flatten the solute profile occurs per unit time. For a given thermal gradient $G$, the undercoolings at $\dot{T}_{1}$ and $\dot{T}_{2}$ can be calculated using Eq. 4 and are represented schematically in Fig. 13(b). The black horizontal dashed line exemplifies a particular undercooling $\Delta T_{n}$ required for IMC nucleation on the most potent nucleant. Nucleation of an IMC on this nucleant is only possible in the region where $\Delta T>\Delta T_{n}$. The difference in the available undercooling between $\dot{T}_{1}$ and $\dot{T}_{2}$ is shaded in Fig. 13 (b), showing that a higher cooling rate only slightly increases the volume of liquid that is sufficiently undercooled to initiate nucleation.

Evidence to support this analysis is shown in Fig. 14(a) that plots the measured

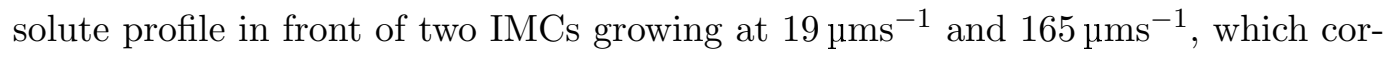
responded to equivalent boundary layer thickness of $300 \mu \mathrm{m}$ and $34 \mu \mathrm{m}$ respectively. Using Eq. 4 and the best estimates for the solidification parameters as previously described, the available undercoolings for the two cases are plotted in Fig. 14(b). The difference in undercooling between these two cases is indicated by an orange dashed curve in Fig. 14(b). By increasing the growth velocity by 9 times from 

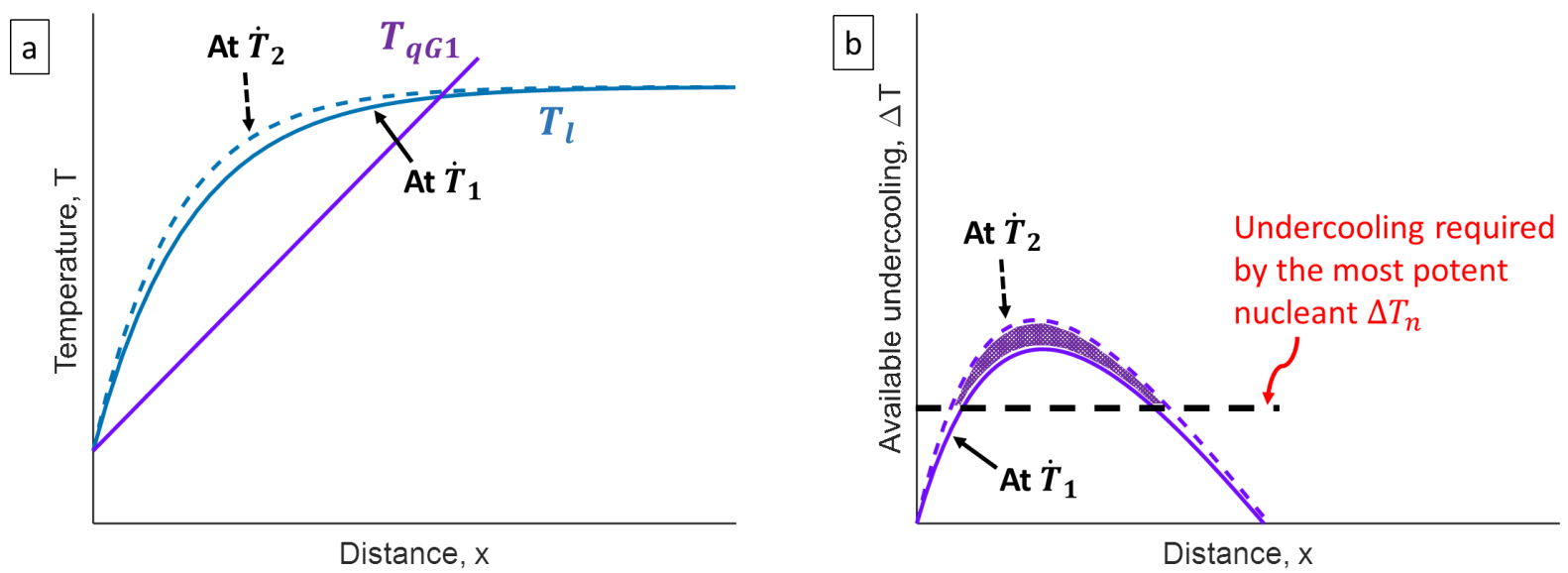

Figure 13: (a) Schematic of the equilibrium liquidus temperature profiles at $\dot{T}_{1}$ and $\dot{T}_{2}\left(\dot{T}_{2}>\dot{T}_{1}\right)$ and thermal gradient $G$. (b) Undercooling curves at $\dot{T}_{1}$ and $\dot{T}_{2}$. The black horizontal dashed line indicates the minimum undercooling required by the most potent nucleant to form a new IMC.

$19 \mathrm{\mu m} \mathrm{s}^{-1}$ to $165 \mathrm{\mu m} \mathrm{s}^{-1}, \Delta T_{\max }$ increased by only $\sim 9 \%$, from $26.5 \mathrm{~K}$ to $29 \mathrm{~K}$, while the $U Z$ remained essentially unchanged at $\sim 6 \mathrm{~mm}$.

In summary, near isothermal solidification showed the highest IMC density at each cooling rate (Fig. 8), because these conditions provided the largest $U Z$ and the largest $\Delta T_{\max }$. Under directional solidification, the IMC density showed a strong dependence on the thermal gradient and a weak sensitivity to cooling rate (Fig. 8), because while both $U Z$ and $\Delta T_{\max }$ were strongly sensitive to thermal gradient, they have been shown analytically to be only weakly affected by cooling rate.

\section{Conclusions}

The formation of primary $\mathrm{Al}_{13} \mathrm{Fe}_{4}$ IMCs and the effect of $\mathrm{TiB}_{2}$ and TiC inoculation during the solidification of a hypereutectic Al-3Fe alloy has been investigated systematically using in-situ laboratory and synchrotron X-ray arrangements. With 4,531 IMCs studied in 56 experiments, the key findings are summarised as follows.

1. $\mathrm{TiB}_{2}$ and $\mathrm{TiC}$ inoculation consistently increased the number density and average formation rate of primary Fe-rich IMCs under all conditions.

2. In vertical, directional cooling, IMCs primarily nucleated on the sample oxide skins in the non-inoculated alloy, but nucleated preferentially on the $\mathrm{TiB}_{2}$ 
(a)

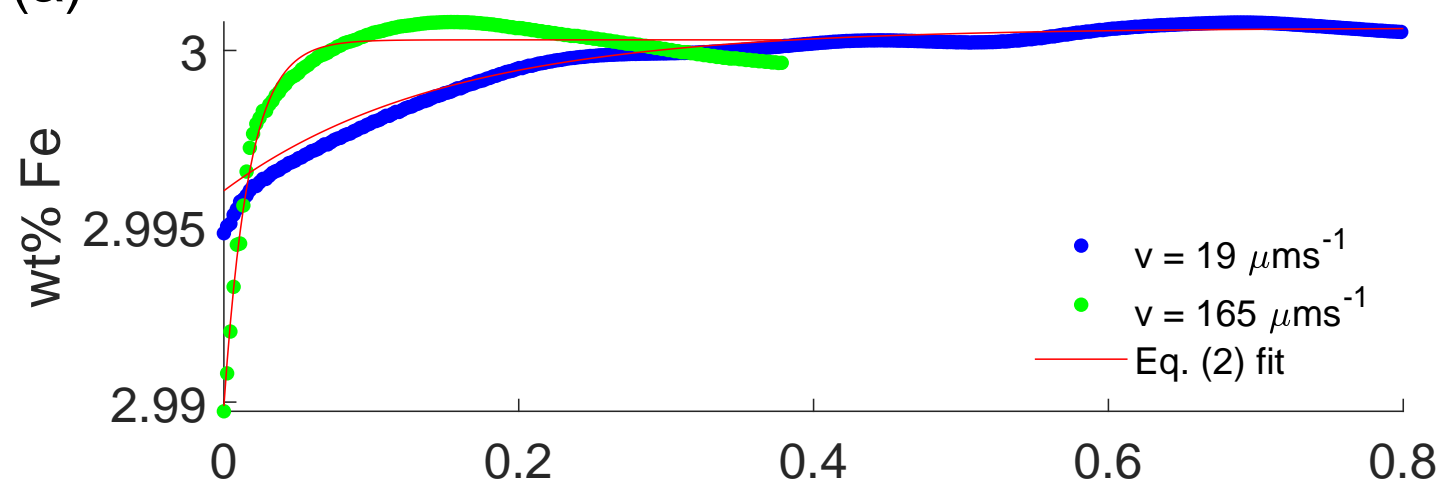

(b)

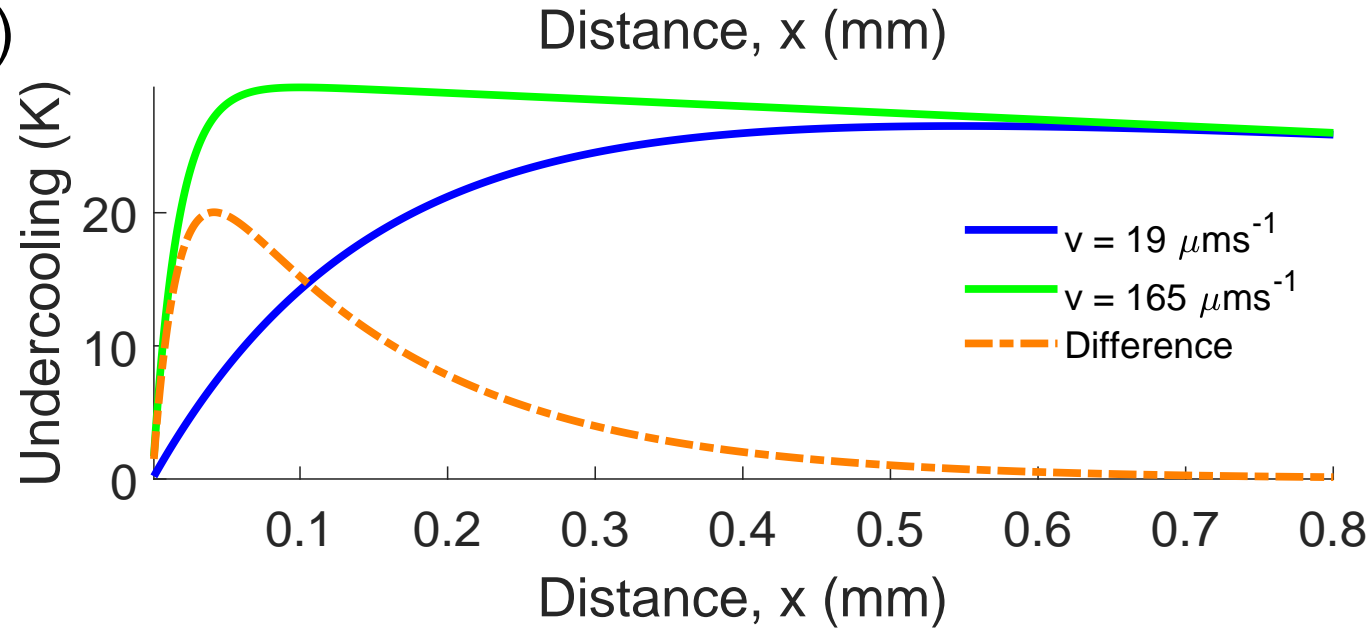

Figure 14: (a) Measured solute profile (wt\% $\%$ e) for two IMC growing at $19 \mu \mathrm{ms}^{-1}$ and $165 \mathrm{\mu ms}^{-1}$. (b) Calculated undercooling distribution in the liquid ahead of the IMC for the profiles shown in (a) using Eq. 4. The orange dashed curve represents the difference in the undercooling for the two cases. The following parameters were used: $\Delta C_{0}=-1 \mathrm{wt} \%, G=5 \mathrm{~K} \mathrm{~mm}^{-1}, D_{l}=2.82 \times 10^{-9} \mathrm{~m}^{2} \mathrm{~s}^{-1}, m=30$ $\mathrm{Kwt} \%^{-1}$.

particles when $\mathrm{TiB}_{2}$ was added.

3. Near isothermal solidification showed the highest IMC number density and average formation rate, and both increased with increasing cooling rate. Under directional solidification, the IMC density was dominated by thermal gradient, and had a weak sensitivity to cooling rate.

4. A model for IMC formation was proposed to explain the experimental findings qualitatively and quantitatively, accounting for the effect of thermal gradient and cooling rate on the volume of the undercooled liquid and the magnitude of the available undercooling. The probability of nucleation was controlled by the size of the undercooled liquid volume and the magnitude of the undercooling 
within this volume, both of which were dominated by thermal gradient.

These findings may have implications for commercial practices to promote conditions that maximise the number of IMC formation events, and so minimise their average size and detrimental effect on alloy ductility and toughness.

\section{Acknowledgements}

This work was supported by EPSRC (UK) under grant number EP/N007638/1 (LiME - Liquid Metal Engineering). This work was enabled by synchrotron beamtime ID 19 at European Synchrotron Radiation Facility (Experiment No. MA4063).

\section{References}

[1] G. Liu, C. E. Bangs, D. B. Muller, Stock dynamics and emission pathways of the global aluminium cycle, Nat. Clim. Chang. 3 (4) (2012) 338-342.

[2] V. Kevorkijan, Challenges and advantages of recycling wrought aluminium alloys from lower grades of metallurgically clean scrap, Mater. Tehnol. 47 (1) (2013) 13-23.

[3] H. W. L. Phillips, Equilibrium diagrams of aluminium alloy systems, London : Aluminium Development Association, 1961.

[4] J. A. Taylor, Iron-Containing Intermetallic Phases in Al-Si Based Casting Alloys, Procedia Mater. Sci. 1 (2012) 19-33.

[5] L. Ceschini, I. Boromei, A. Morri, S. Seifeddine, I. L. Svensson, Microstructure, tensile and fatigue properties of the $\mathrm{Al}-10 \% \mathrm{Si}-2 \% \mathrm{Cu}$ alloy with different $\mathrm{Fe}$ and Mn content cast under controlled conditions, J. Mater. Process. Technol. 209 (15-16) (2009) 5669-5679.

[6] S. Ji, W. Yang, F. Gao, D. Watson, Z. Fan, Effect of Iron in Al-Mg-Si-Mn Ductile Diecast Alloy, Light Met. 2013 (2013). 
[7] J. Cui, H. J. Roven, Recycling of automotive aluminum, Trans. Nonferrous Met. Soc. China (English Ed.) 20 (11) (2010) 2057-2063.

[8] D. Paraskevas, K. Kellens, A. Van de Voorde, W. Dewulf, J. R. Duflou, Environmental Impact Analysis of Primary Aluminium Production at Country Level, Procedia CIRP 40 (2016) 209-213.

[9] L. A. Narayanan, F. H. Samuel, J. E. Gruzleski, Dissolution of iron intermetallics in Al-Si Alloys through nonequilibrium heat treatment, Metall. Mater. Trans. A 26 (8) (1995) 2161-2174.

[10] B. Lin, W. Zhang, Y. Zhao, Y. Li, Solid-state transformation of Fe-rich intermetallic phases in $\mathrm{Al}-5.0 \mathrm{Cu}-0.6 \mathrm{Mn}$ squeeze cast alloy with variable Fe contents during solution heat treatment, Mater. Charact. 104 (2015) 124-131.

[11] L. Backerud, Kinetic Aspects of the solidification of Binary and Ternary Alloy Systems, jernkontorets Ann 152 (3) (1968) 109.

[12] L. F. Mondolfo, Aluminum alloys : structure and properties, Butterworths, 1976.

[13] L. Backerud, E. Krol, J. Tamminen, Solidification characteristics of aluminium alloys, Skanaluminium, Universitetsforlaget AS, 1986.

[14] C. M. Allen, K. A. Q. O'Reilly, B. Cantor, P. V. Evans, Intermetallic phase selection in 1XXX Al alloys, Prog. Mater. Sci. 43 (2) (1998) 89-170.

[15] Y. Wang, H. Jones, P. Evans, Eutectic solidification characteristics of Bridgman grown AI-3Fe-0.1V alloy, J. Mater. Sci. 33 (21) (1998) 5205-5220.

[16] Y. Wang, H. Jones, Effect of growth velocity on the growth temperature of the Al-AlxFe eutectic in Al-2.85Fe-0.12V alloy, Metall. Mater. Trans. A 32 (5) (2001) 1251-1253. 
[17] G. Sha, K. O'Reilly, B. Cantor, J. Worth, R. Hamerton, Growth related metastable phase selection in a 6xxx series wrought Al alloy, Mater. Sci. Eng. A 304-306 (2001) 612-616.

[18] W. Khalifa, F. H. Samuel, J. E. Gruzleski, H. W. Doty, S. Valtierra, Nucleation of Fe-intermetallic phases in the Al-Si-Fe alloys, Metall. Mater. Trans. A 36 (4) (2005) 1017-1032.

[19] H. Jones, Some effects of solidification kinetics on microstructure formation in aluminium-base alloys, Mater. Sci. Eng. A 413-414 (2005) 165-173.

[20] G. Sha, K. O'Reilly, B. Cantor, Characterization of Fe-Rich Intermetallic Phases in a 6xxx Series Al Alloy, Mater. Sci. Forum 519-521 (2006) 1721-1726.

[21] S. Kumar, K. A. Q. O'Reilly, Influence of Al grain structure on Fe bearing intermetallics during DC casting of an Al-Mg-Si alloy, Mater. Charact. 120 (2016) 311-322.

[22] S. Kumar, P. S. Grant, K. A. Q. O'Reilly, Evolution of Fe Bearing Intermetallics During DC Casting and Homogenization of an Al-Mg-Si Al Alloy, Metall. Mater. Trans. A 47 (6) (2016) 3000-3014.

[23] A. M. Samuel, A. Pennors, C. Villeneuve, F. H. Samuel, H. W. Doty, S. Valtierra, Effect of cooling rate and Sr-modification on porosity and Feintermetallics formation in Al-6.5\% Si-3.5\% Cu-Fe alloys, Int. J. Cast Met. Res. 13 (4) (2000) 231-253.

[24] L. Lu, A. K. Dahle, Iron-rich intermetallic phases and their role in casting defect formation in hypoeutectic Al-Si alloys, Metall. Mater. Trans. A 36 (13) (2005) 819-835.

[25] X. Cao, J. Campbell, The nucleation of Fe-Rich phases on oxide films in Al11.5Si-0.4Mg cast alloys, Metall. Mater. Trans. A 34 (7) (2003) 1409-1420. 
[26] X. Cao, J. Campbell, The solidification characteristics of Fe-rich intermetallics in Al-11.5Si-0.4Mg cast alloys, Metall. Mater. Trans. A 35 (5) (2004) 14251435.

[27] D. N. Miller, L. Lu, A. K. Dahle, The role of oxides in the formation of primary iron intermetallics in an Al-11.6Si-0.37Mg alloy, Metall. Mater. Trans. B Process Metall. Mater. Process. Sci. 37 (6) (2006) 873-878.

[28] L. A. Narayanan, F. H. Samuel, J. E. Gruzleski, Crystallization behavior of iron-containing intermetallic compounds in 319 aluminum alloy, Metall. Mater. Trans. A 25 (8) (1994) 1761-1773.

[29] A. Lui, P. S. Grant, I. C. Stone, K. A. Q. O'Reilly, The Role of Grain Refiner in the Nucleation of AlFeSi Intermetallic Phases During Solidification of a 6xxx Aluminum Alloy, Metall. Mater. Trans. A 50 (11) (2019) 5242-5252.

[30] S. Terzi, J. A. Taylor, Y. H. Cho, L. Salvo, M. Suery, E. Boller, A. K. Dahle, In situ study of nucleation and growth of the irregular $\alpha-\mathrm{Al} / \beta-\mathrm{Al} 5 \mathrm{FeSi}$ eutectic by 3-D synchrotron X-ray microtomography, Acta Mater. 58 (16) (2010) 53705380.

[31] C. Puncreobutr, A. B. Phillion, J. L. Fife, P. Rockett, A. P. Horsfield, P. D. Lee, In situ quantification of the nucleation and growth of Fe-rich intermetallics during Al alloy solidification, Acta Mater. 79 (2014) 292-303.

[32] J. Wang, P. D. Lee, R. W. Hamilton, M. Li, J. Allison, The kinetics of Fe-rich intermetallic formation in aluminium alloys: In situ observation, Scr. Mater. 60 (7) (2009) 516-519.

[33] B. Kim, S. Lee, S. Lee, H. Yasuda, Real-Time Radiographic Observation of Solidification Behavior of $\mathrm{Al}-\mathrm{Si}-\mathrm{Cu}$ Casting Alloys with the Variation of Iron Content, Mater. Trans. 53 (2) (2012) 374-379.

[34] A. Bjurenstedt, D. Casari, S. Seifeddine, R. H. Mathiesen, A. K. Dahle, In-situ 
study of morphology and growth of primary $\alpha-\mathrm{Al}(\mathrm{FeMnCr}) \mathrm{Si}$ intermetallics in an Al-Si alloy, Acta Mater. 130 (2017) 1-9.

[35] S. Feng, E. Liotti, A. Lui, S. Kumar, A. Mahadevegowda, K. A. Q. O'Reilly,

[40] A. G. Murphy, W. U. Mirihanage, D. J. Browne, R. H. Mathiesen, Equiaxed dendritic solidification and grain refiner potency characterised through in situ X-radiography, Acta Mater. 95 (2015) 83-89. 
[41] Y. Xu, D. Casari, Q. Du, R. H. Mathiesen, L. Arnberg, Y. Li, Heterogeneous nucleation and grain growth of inoculated aluminium alloys: An integrated study by in-situ X-radiography and numerical modelling, Acta Mater. 140 (2017) 224-239.

[42] Y. Xu, D. Casari, R. H. Mathiesen, Y. Li, Revealing the heterogeneous nucleation behavior of equiaxed grains of inoculated $\mathrm{Al}$ alloys during directional solidification, Acta Mater. 149 (2018) 312-325.

[43] K. Liu, X. Cao, X. G. Chen, Solidification of iron-rich intermetallic phases in Al-4.5Cu-0.3Fe cast alloy, Metall. Mater. Trans. A Phys. Metall. Mater. Sci. 42 (7) (2011) 2004-2016.

[44] G. Sha, K. O'Reilly, B. Cantor, R. Hamerton, J. Worth, Effect of Grain Refiner on Intermetallic Phase Formation in Directional Solidification of 6xxx Series Wrought Al Alloys, Mater. Sci. Forum 331-337 (2000) 253-258.

[45] J. Rakhmonov, G. Timelli, F. Bonollo, Influence of Melt Superheat, Sr Modifier, and Al-5Ti-1B Grain Refiner on Microstructural Evolution of Secondary Al-Si-Cu Alloys, Metall. Mater. Trans. A Phys. Metall. Mater. Sci. 47 (11) (2016) 5510-5521.

[46] A. L. Greer, A. M. Bunn, A. Tronche, P. V. Evans, D. J. Bristow, Modelling of inoculation of metallic melts: application to grain refinement of aluminium by Al-Ti-B, Acta Mater. 48 (11) (2000) 2823-2835.

[47] A. Prasad, L. Yuan, P. D. Lee, M. Easton, D. StJohn, The effect of the melt thermal gradient on the size of the constitutionally supercooled zone, IOP Conf. Ser. Mater. Sci. Eng. 117 (1) (2016).

[48] E. Liotti, A. Lui, S. Kumar, Z. Guo, C. Bi, T. Connolley, P. S. Grant, The spatial and temporal distribution of dendrite fragmentation in solidifying $\mathrm{Al}-\mathrm{Cu}$ alloys under different conditions, Acta Mater. 121 (2016) 384-395. 

nucleation in metallic alloys using x-ray radiography and machine learning, Sci. Adv. 4 (4) (2018) eaar4004.

[50] W. Tiller, K. Jackson, J. Rutter, B. Chalmers, The redistribution of solute atoms during the solidification of metals, Acta Metall. 1 (4) (1953) 428-437. redistribution during solidification, Can. J. Phys. 33 (12) (1955) 723-745.

[52] Y. Du, Y. Chang, B. Huang, W. Gong, Z. Jin, H. Xu, Z. Yuan, Y. Liu, Y. He, F.-Y. Xie, Diffusion coefficients of some solutes in fcc and liquid Al: critical evaluation and correlation, Mater. Sci. Eng. A 363 (1-2) (2003) 140-151. function, Advances in Computational Mathematics 5 (1996) 329-359.

[54] Z. P. Que, Y. P. Zhou, Y. Wang, Z. Fan, Composition templating for heterogeneous nucleation of intermetailic compounds, in: Proc. of the 6th Decennial Intern. Conf. on Solidification Process. at Old Windsor, UK, 2017. 